\title{
COMPARATIVE STUDY AMONG DIRECT DESIGN METHOD, EQUIVALENT FRAME METHOD AND FINITE FRAME METHOD FOR ANALYSIS OF SLABS
}

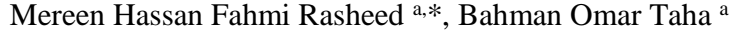 \\ ${ }^{\text {a }}$ Erbil Engineering Technical College, University of Erbil Polytechnic, Erbil, Kurdistan Region, Iraq - (mereen.akrawi, \\ bahman.omar)@epu.edu.krd
}

\begin{abstract}
:
Beam-column frame system and flat plate slab system are analysed by semi-empirical, Direct Design method (DDM) and approximate elastic method, Equivalent Frame method (EFM) and the results of both methods are compared with computer software based on Finite Element method (FEM), taking into account the effect of changing the beam and column stiffness and the panel length ratio, for 3, 4, and 5 equal span frames and three non-equal spans. The moment coefficients with respect to the maximum clear span moment are determined by the three methods for negative end moments at the face of support and mid span positive moment. These coefficients are constant in DDM, while in EFM are changed with changing the column and beam sizes. The results of EFM is more accurate than DDM, on the bases of results of EFM, new moment coefficients are suggested to use instead of DDM moment coefficients. In case of EFM calculation aren't satisfactory for hand calculations the FEM is used by applying available computer software.
\end{abstract}

KEYWORDS: Direct Design Method, Equivalent Frame Method, Finite Element Method.

\section{INTRODUCTION AND METHODOLOGY}

\subsection{Introduction}

Slabs are usually used to provide useful surfaces, such as roofs, floors in reinforced concrete buildings and are usually horizontal, in some times maybe vertical such as in water tanks and retaining walls. Slabs may be supported by masonry or reinforced concrete walls or beams, or directly supported by columns which is called flat plate slabs. Also, slabs may have supported on four edges or two opposite sides. In slabs which is supported on four sides, the slabs act in two direction and main reinforcements are used in two directions, while in slabs supported on two opposite sides, the slab acts in one direction and main reinforcement is used in one direction (short span) and secondary reinforcement is used in other direction (long span).

In flat slabs, shear stresses are critical, and the slab should be designed to resist shear stresses. Column capital and drop panels may be used in flat slabs to increase the shear resistance of the slab.

Different methods are used to design the slabs supported by beams, flat slabs, flat plate slabs and two-way joist slabs, methods 3 of the 1963 ACI Code may be used to design the slab [1], also, can be used for slabs supported by walls, steel beams, or monolithic concrete beams having total depth greater than 3 times the slab thickness. Semi empirical method (Direct Design Method (DDM)) and an approximate elastic analysis method (Equivalent Frame Method) are used for the analysis and design of slabs [2,3].

In both methods, the slab is divided into middle strip and column strip of width equal to one-fourth of the smaller of the panel dimensions (L1 \& L2), where (L1) is the span in the direction of the analysis and (L2) is the perpendicular span of the slab panel.

\subsection{REVIEW OF LITERATURES}

(Patel and Dubey, 2016); presented a comparative study between Direct Design Method (DDM) and Equivalent Frame method (EFM) and then compared with the Finite Element Method (FEM) for the flat slabs. They concluded that (EFM) is more accurate than (DDM), but it's not satisfactory for hand calculations, therefore they recommended using of computer software based on (FEM).

(Patel and Sigi, 2014); presented the analysis of flat slabs by various codes (IS 456-2000, ACI 380-08, BS 8110-1997) and compared with the finite element analysis by computer software (SAFE). The equivalent frame method is used by different codes to determine the column strip and middle strip moments and then compared with the FEM results using software (SAFE).

(Hassan and Ahmed, 2016); presented a comparison study of the analysis of flat slab by two methods given by ACI 318-05 Code, DDM and EFM, they concluded that the exterior negative moment obtained by (EFM) is greater than (DDM), also (DDM) has many limitations and based on coefficients which are not changed by changing beam thickness, while (FEM) have no limitations and the beam and column stiffness are taken into consideration and calculation of column and middle strip moments.

(Kndama,2015); presented a study on flat plate slab system analysed using (EFM). Its concluded that two-dimensional (EFM) that consider nonlinearity effects is effective in determining slab moments only, while three-dimensional (EFM) underestimate slab and column moments.

In this study different numerical example are solved using DDM, EFM and FEM using STAADPRO computer software, the effect of the beam and column dimensions and stiffness are considered. The results of the three methods are compared for three, four and five equal spans for slab system with beams and flat slabs with and without edge beams.

Also, comparison between these methods are performed for slab system panels with different length values.

\footnotetext{
* Corresponding author
} 


\subsection{Methodology}

ACI code and other codes suggest any of the two methods Direct Design Method (DDM) and Equivalent Frame Method (EFM). (DDM) has many restrictions, while (EFM) has no restrictions and not satisfactory for hand calculations.

The moment at exterior and interior support are determined by both DDM \& EFM and compared with the results of (FEM) using computer (STAAD Pro).

The following variable are taken into consideration.

1- Number of spans (3,4 and 5) equal spans.

2- For beam-column system; the dimensions and stiffness the beam and columns are considered. Beams $(500 \times 700)$, (400x600), (3300x500). Columns (500x500), (400x400), (300x300).

3- For flat plate slab system; the analysis is performed for flat slab with and without edge beams.

4- Different span lengths (3, $6 \& 12 \mathrm{~m})$.

5- Different $\left(\frac{L_{2}}{L_{1}}\right)$ ratios $(0.5,1 \& 2)$.

6- Different $\left(\psi=\frac{K_{B}}{K_{C}}\right)$ ratios of beam stiffness / column stiffness.

\section{ANALYSIS}

Different beam-column frames are analyzed by three methods, Direct Design Method (DDM), Equivalent Frame Method (EFM) and Finite Element Method (FEM) using computer software (STAAD Pro). End moments and midspan moments are determined as a ratio with respect to the total net moments Mo,

$$
M_{o}=\frac{W_{u} \cdot L_{2} \cdot L_{n 1}^{2}}{8}
$$

Where: $\mathrm{Mo}=$ Total net moments

$\mathrm{Wu}$ : Ultimate factored load

L2: Perpendicular span of the slab panel

Ln1: Clear span in the direction of the analysis

The results of the three methods are compared to show the safe moment coefficients compares with DDM coefficients. All the problems are analyzed for the following data

Cylindrical concrete strength $\left(\mathrm{fc}^{\prime}\right)=28 \mathrm{MPa}$

Steel yield $(\mathrm{fy})=414 \mathrm{MPa}$

Live load (L.L) $=5 \mathrm{kN} / \mathrm{m} 2$

Perpendicular panel direction $\mathrm{L} 2=6 \mathrm{~m}$

Problem 1: - Three equal spans of $\mathrm{L} 1=6 \mathrm{~m}$

Column dimensions $(500 \times 500) \mathrm{mm}$

Beam dimensions $(500 \times 700) \mathrm{mm}$

The analysis is repeated for $\mathrm{L} 1=3 \mathrm{~m}$ and $\mathrm{L} 1=12 \mathrm{~m}$

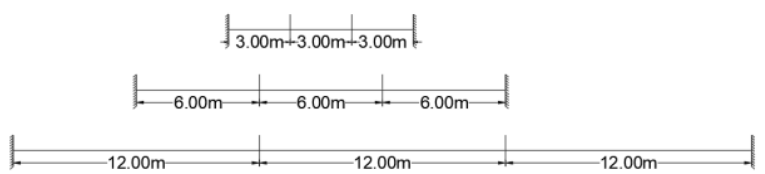

Problem 2: - Three equal spans of $\mathrm{L} 1=6 \mathrm{~m}$, the external end support is simply supported.

Column dimensions $(500 \times 500) \mathrm{mm}$

Beam dimensions $(500 \times 700) \mathrm{mm}$

The analysis is repeated for $\mathrm{L} 1=3 \mathrm{~m}$ and $\mathrm{L} 1=12 \mathrm{~m}$

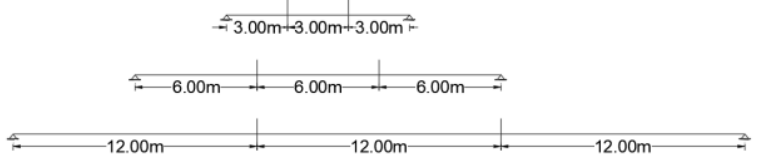

Problem 3: - Three equal spans of $\mathrm{L} 1=6 \mathrm{~m}$

Column dimensions $(400 \times 400) \mathrm{mm}$

Beam dimensions (400x600) $\mathrm{mm}$

The analysis is repeated for $\mathrm{L} 1=3 \mathrm{~m}$ and $\mathrm{L} 1=12 \mathrm{~m}$

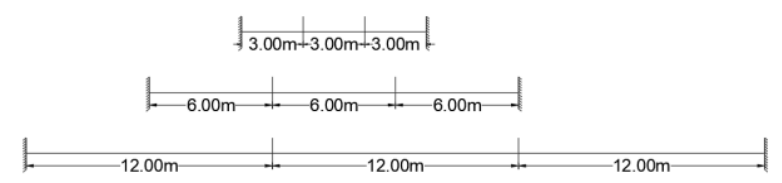

Problem 4: - Three equal spans of L1 $=6 \mathrm{~m}$, the external end support is simply supported.

Column dimensions $(400 \times 400) \mathrm{mm}$

Beam dimensions $(400 \times 600) \mathrm{mm}$

The analysis is repeated for $L 1=3 \mathrm{~m}$ and $\mathrm{L} 1=12 \mathrm{~m}$

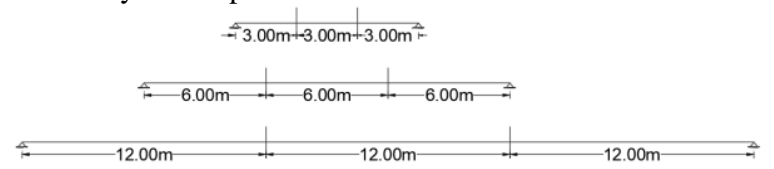

Problem 5: - Three equal spans of $(\mathrm{L} 1=6 \mathrm{~m})$

column dimension $(300 \times 300) \mathrm{mm}$

and beam dimensions $(300 \times 500) \mathrm{mm}$

Also, the analysis is performed for $(\mathrm{L} 1=3 \mathrm{~m} \& \mathrm{~L} 1=12 \mathrm{~m})$

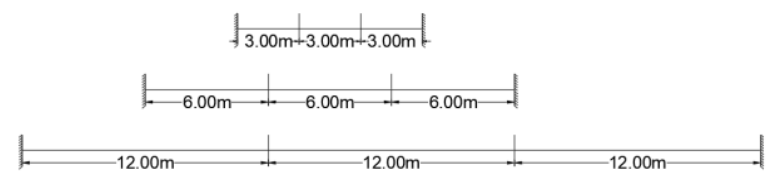

Problem 6: - Same as problem 5, but the external end or support is simply supported

All the above problems are analysed by (DDM), (EFM) and (FEM), the result of the analysis is summarized in the following tables (1 to 12).

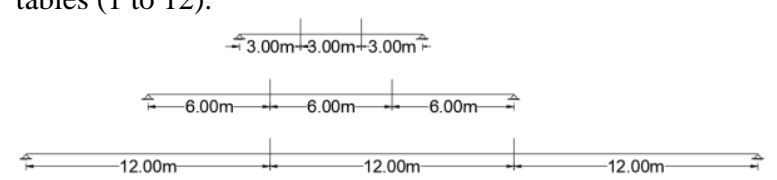

Problem 7: - Three equal span flat plate slabs frame system with thickness $(200 \mathrm{~mm})$ for panel width $\mathrm{L} 2=6.0 \mathrm{~m} \&$ span length $\mathrm{L} 1=6 \mathrm{~m}$, and

column dimensions $(500 \times 500) \mathrm{mm}$

the analysis is performed for two cases:

1- Flat slab without edge beams at external panel.

2- Flat slab with edge beams at external panel Beams dimension (500x700).

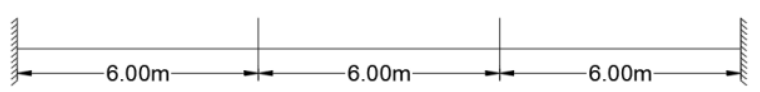

Problem 8: - Four equal span flat plate slabs frame for the same data of problem (7).

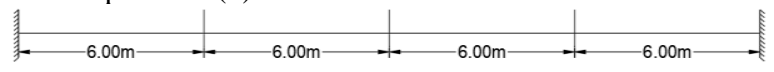

Problem 9: - Five equal span flat plate slabs frame for the same data of problem (7).

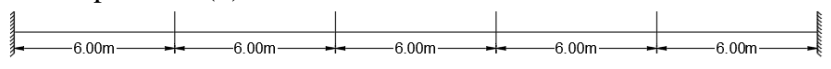

Problem 10: - Three spans frame, the middle span is less than the external spans by $(33 \%)$. L2 $=6 \mathrm{~m}$

Column dimensions $(500 \times 500) \mathrm{mm}$

Beam dimensions $(500 \times 700) \mathrm{mm}$

Slab thickness $=200 \mathrm{~mm}$

The analysis is performed fir two cases:

$$
\begin{aligned}
& \text { 1- Beam-Column system. } \\
& 2-\quad \text { Flat plate slab system. }
\end{aligned}
$$

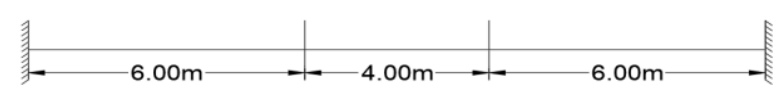

Problem 11: - Same as problem (10) but the external support is simply supported

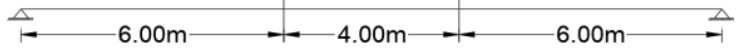


Problem 12 \& 13: - Same as problem (10 \&11), the middle span is greater than the external or outer spans by $33 \%$.

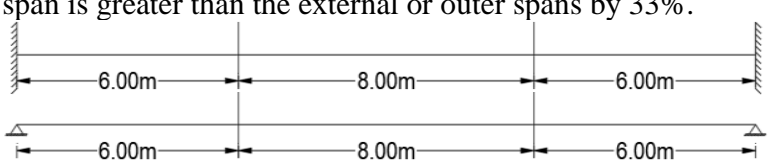

Problem 14: - Four equal span beam- column frame system, $\mathrm{L} 2=6 \mathrm{~m}, \mathrm{~L} 1=6 \mathrm{~m}, \mathrm{~L} 2 / \mathrm{L} 1=1.0$

Column dimensions (500x500) $\mathrm{mm}$ and beam dimensions (500x700) $\mathrm{mm}$

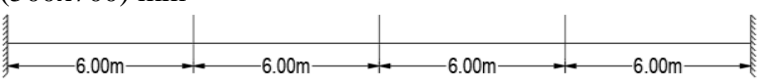

Problem 15: - Four equal span beam- column frame system the external support is simply supported, L2 $=6 \mathrm{~m}, \mathrm{~L} 1=6 \mathrm{~m}$, $\mathrm{L} 2 / \mathrm{L} 1=1.0$
Column dimensions $(500 \times 500) \mathrm{mm}$ and beam dimensions (500x700) mm

Problem 16: - Five equal span beam- column frame system, for the same above data

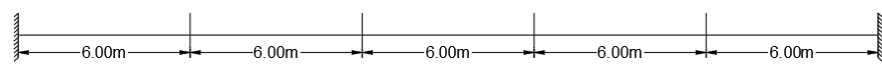

Problem 17: - Five equal span beam- column frame system the external support is simply supported.

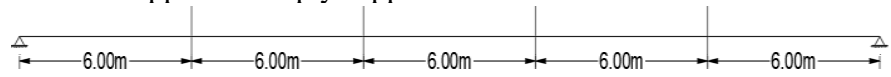

Notes:

-The problems are symmetric moments of spans until center lines are shown in the tables

- $\mathrm{M}=\alpha \mathrm{Mo}$, where $\alpha$ is the moment coefficient \& $\psi=$ Beam stiffness/Column stiffness

Table 1. End moments at face of support and midspan moments determined by three methods DDM, EFM \& FEM. Column dimension (500x500) $\mathrm{mm} \&$ beam dimension $(500 \times 700) \mathrm{mm}$.

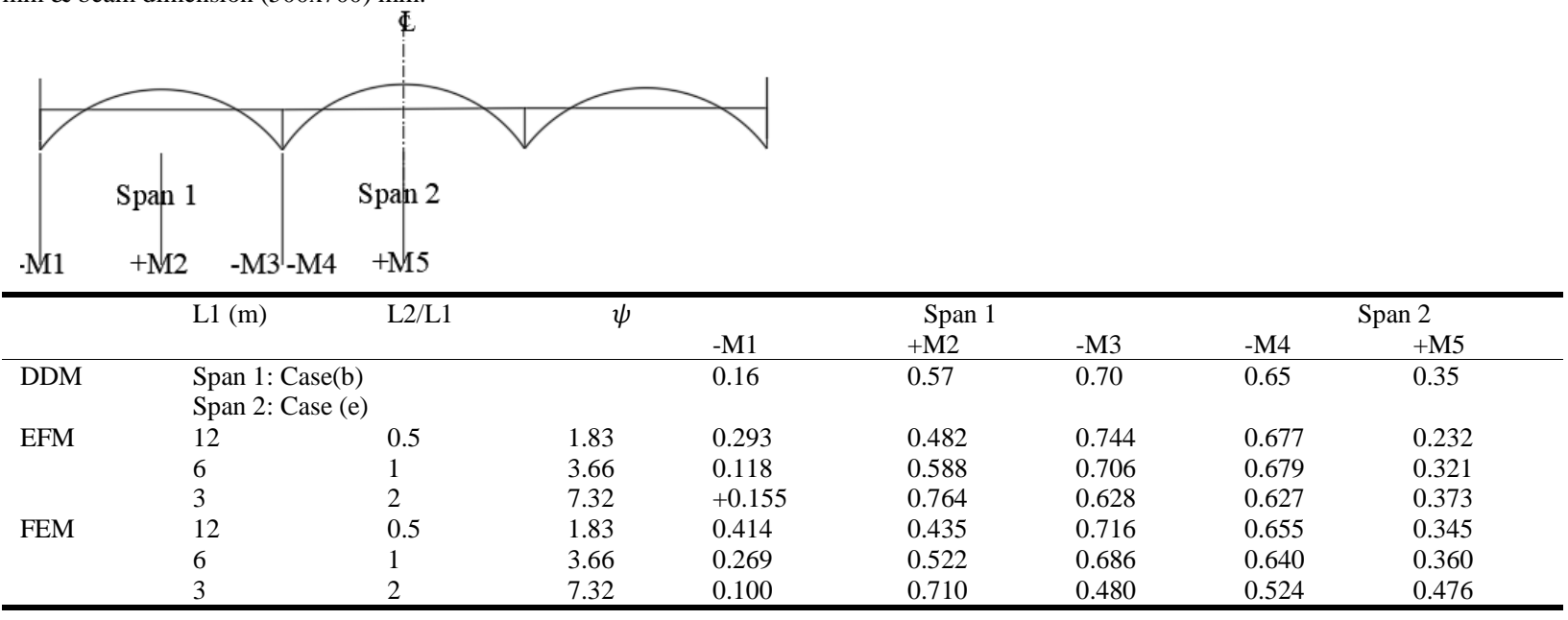

Table 2. End and midspan moments coefficients for the frame with external support simply supported. Column dimension (500x500) $\mathrm{mm} \&$ beam dimension $(500 \times 700) \mathrm{mm}$

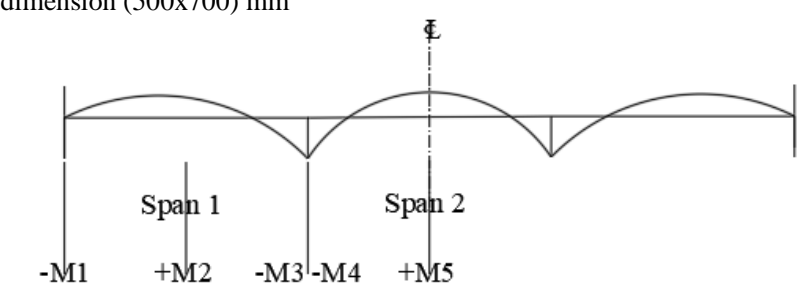

\begin{tabular}{lllcccccc}
\hline & L1 $(\mathrm{m})$ & L2/L1 & $\psi$ & \multicolumn{2}{c}{ Span 1 } & \multicolumn{3}{c}{ Span 2 } \\
& & & & $-\mathrm{M} 1$ & $+\mathrm{M} 2$ & $-\mathrm{M} 3$ & $-\mathrm{M} 4$ & \\
\hline DDM & Span 1: Case(a) & & 0 & 0.63 & 0.75 & 0.65 & 0.35 \\
& Span 2: Case (e) & & & & & & & \\
EFM & 12 & 0.5 & 1.83 & 0 & 0.572 & 0.856 & 0.720 & 0.280 \\
& 6 & 1 & 3.66 & 0 & 0.620 & 0.76 & 0.720 & 0.280 \\
& 3 & 2 & 7.32 & 0 & 0.671 & 0.657 & 0.661 & 0.339 \\
FEM & 12 & 0.5 & 1.83 & 0 & 0.571 & 0.858 & 0.715 & 0.285 \\
& 6 & 1 & 3.66 & 0 & 0.617 & 0.766 & 0.699 & 0.301 \\
& 3 & 2 & 7.32 & 0 & 0.752 & 0.497 & 0.540 & 0.460 \\
\hline
\end{tabular}

Table 3. End and midspan moments coefficients for the frame with column dimension (400x400) mm \& beam dimension (400x600) $\mathrm{mm}$

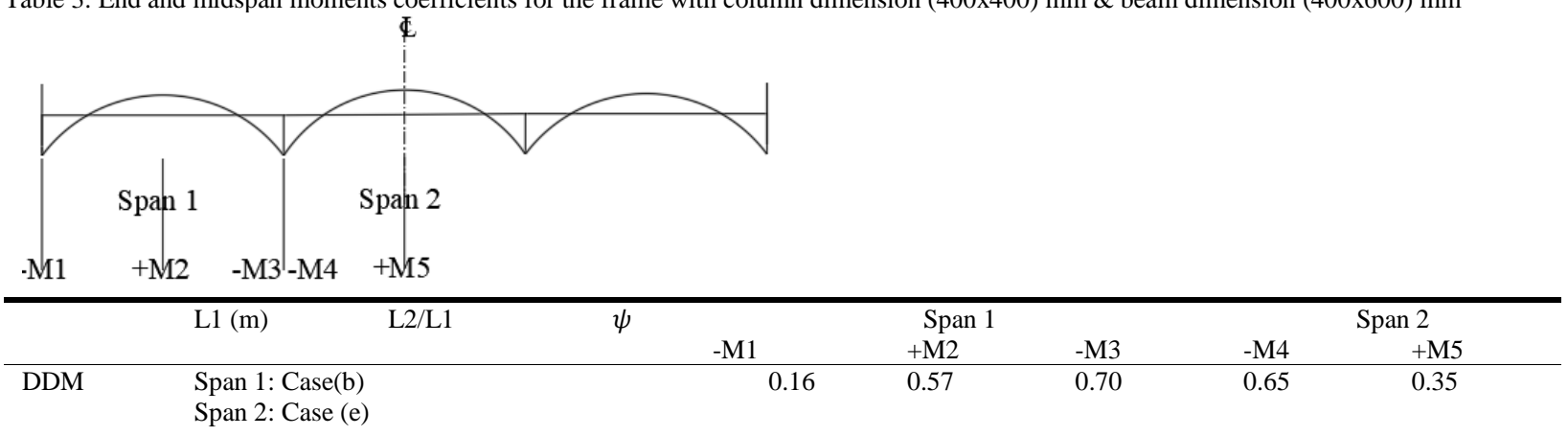




\begin{tabular}{llllllllll} 
EFM & 12 & 0.5 & 2.25 & 0.271 & 0.487 & 0.755 & 0.690 & 0.31 \\
& 6 & 1 & 4.50 & 0.111 & 0.575 & 0.738 & 0.698 & 0.302 \\
\multirow{3}{*}{ FEM } & 3 & 2 & 9.00 & +0.107 & 0.718 & 0.670 & 0.669 & 0.331 \\
& 12 & 0.5 & 2.25 & 0.394 & 0.438 & 0.730 & 0.666 & 0.334 \\
& 6 & 1 & 4.50 & 0.252 & 0.578 & 0.712 & 0.660 & 0.340 \\
& 3 & 2 & 9.00 & 0.076 & 0.672 & 0.580 & 0.584 & 0.416 \\
\hline
\end{tabular}

Table 4. End and midspan moments coefficients for the frame with external support simply supported, column dimension (400x400) mm \& beam dimension $(400 \times 600) \mathrm{mm}$

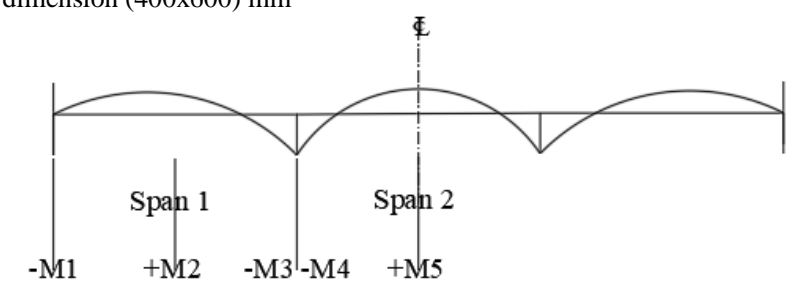

\begin{tabular}{|c|c|c|c|c|c|c|c|c|}
\hline & \multirow{2}{*}{ L1 (m) } & \multirow{2}{*}{$\overline{\mathrm{L} 2 / \mathrm{L} 1}$} & \multirow[t]{2}{*}{$\psi$} & \multicolumn{3}{|c|}{ Span 1} & \multicolumn{2}{|c|}{ Span 2} \\
\hline & & & & -M1 & $+\mathrm{M} 2$ & -M3 & -M4 & +M5 \\
\hline DDM & \multicolumn{2}{|l|}{$\begin{array}{l}\text { Span 1: Case(a) } \\
\text { Span 2: Case (e) }\end{array}$} & & 0 & 0.63 & 0.75 & 0.65 & 0.35 \\
\hline \multirow[t]{3}{*}{ EFM } & 12 & 0.5 & 2.25 & 0 & 0.574 & 0.852 & 0.730 & 0.270 \\
\hline & 6 & 1 & 4.50 & 0 & 0.604 & 0.792 & 0.734 & 0.266 \\
\hline & 3 & 2 & 9.00 & 0 & 0.652 & 0.696 & 0.697 & 0.303 \\
\hline \multirow[t]{3}{*}{ FEM } & 12 & 0.5 & 2.25 & 0 & 0.572 & 0.856 & 0.727 & 0.273 \\
\hline & 6 & 1 & 4.50 & 0 & 0.610 & 0.780 & 0.722 & 0.278 \\
\hline & 3 & 2 & 9.00 & 0 & 0.710 & 0.580 & 0.607 & 0.393 \\
\hline
\end{tabular}

Table 5. End and midspan moments coefficients for the frame with column dimension (300x300) mm \& beam dimension (300x500) mm

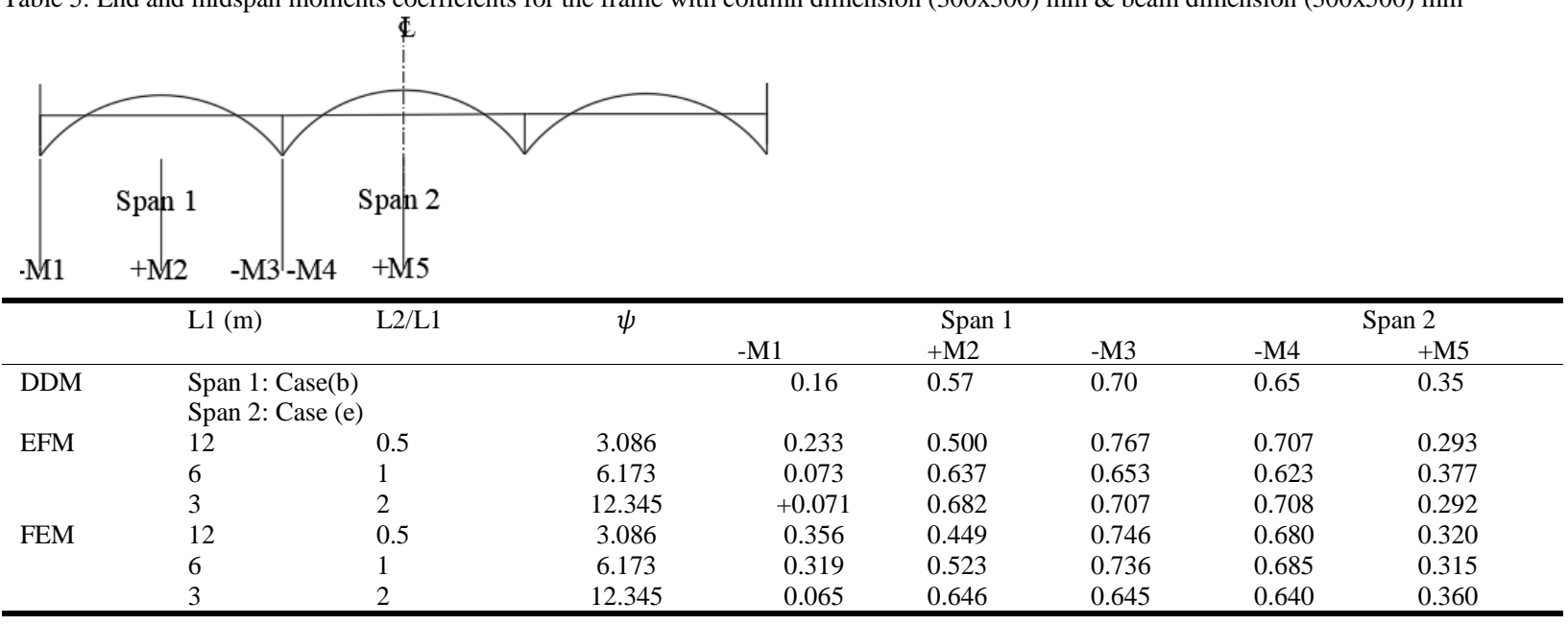

Table 6. End and midspan moments coefficients for the frame with external support simply supported, column dimension (300x300) mm \& beam dimension $(300 \times 500) \mathrm{mm}$

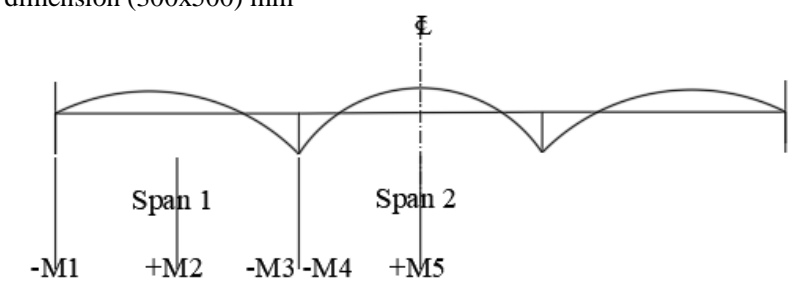

\begin{tabular}{|c|c|c|c|c|c|c|c|c|}
\hline & \multirow{2}{*}{$\mathrm{L} 12(\mathrm{~m})$} & \multirow[t]{2}{*}{ L2/L1 } & \multirow{2}{*}{$\psi$} & \multicolumn{3}{|c|}{ Span 1} & \multicolumn{2}{|c|}{ Span 2} \\
\hline & & & & -M1 & $+\mathrm{M} 2$ & -M3 & -M4 & +M5 \\
\hline DDM & $\begin{array}{l}\text { Span 1: Case(a) } \\
\text { Span 2: Case (e) }\end{array}$ & & & 0 & 0.63 & 0.75 & 0.65 & 0.35 \\
\hline \multirow[t]{3}{*}{ EFM } & 12 & 0.5 & 3.086 & 0 & 0.577 & 0.845 & 0.745 & 0.255 \\
\hline & 6 & 1 & 6.173 & 0 & 0.655 & 0.690 & 0.650 & 0.350 \\
\hline & 3 & 2 & 12.345 & 0 & 0.636 & 0.728 & 0.730 & 0.270 \\
\hline \multirow[t]{3}{*}{ FEM } & 12 & 0.5 & 3.086 & 0 & 0.576 & 0.848 & 0.741 & 0.259 \\
\hline & 6 & 1 & 6.173 & 0 & 0.626 & 0.748 & 0.703 & 0.297 \\
\hline & 3 & 2 & 12.345 & 0 & 0.676 & 0.648 & 0.663 & 0.337 \\
\hline
\end{tabular}


Table 7. End and midspan moments coefficients for the frame $\mathrm{L} 1=12.0 \mathrm{~m}, \mathrm{~L} 2=6.0 \mathrm{~m} \mathrm{~L} 2 / \mathrm{L} 1=0.5$

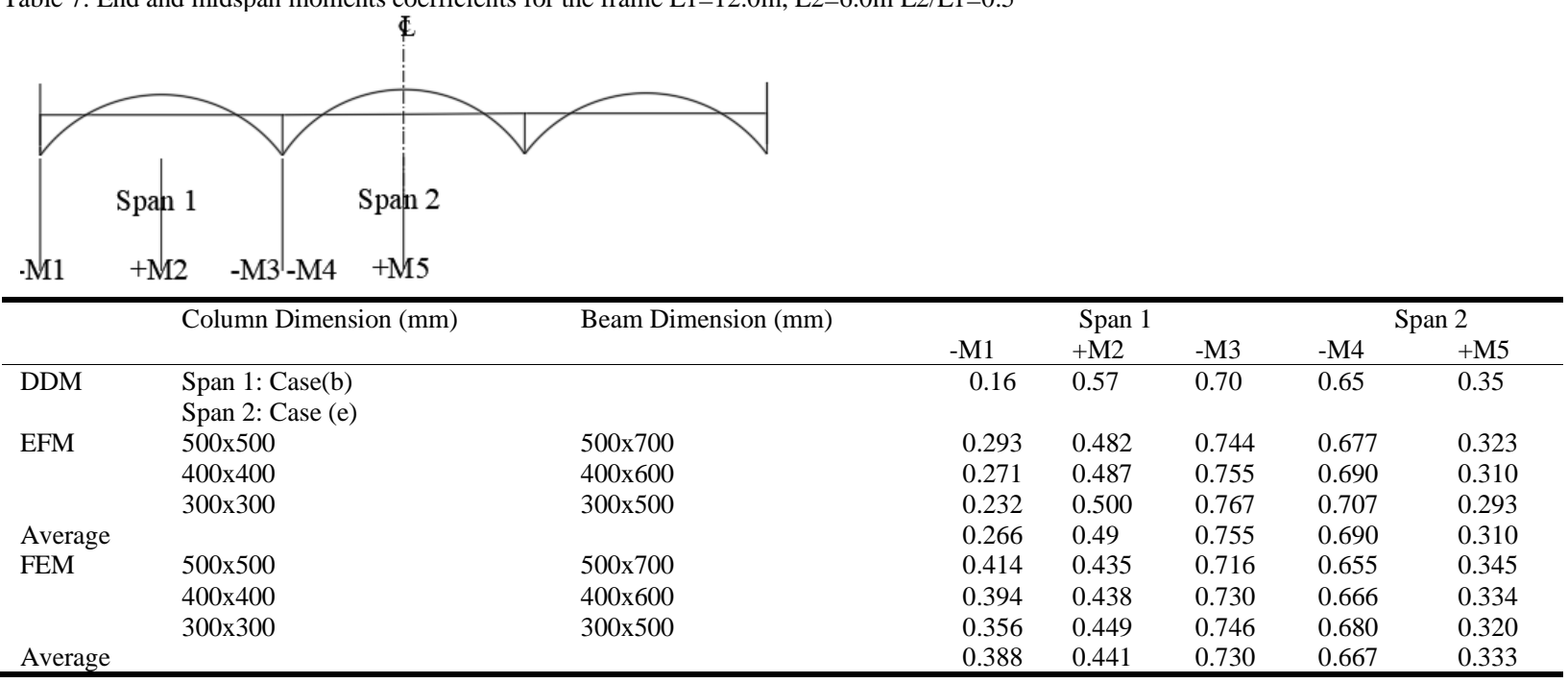

Table 8. End and midspan moments coefficients for frame with simply supported external support, $\mathrm{L} 1=12.0 \mathrm{~m}, \mathrm{~L} 2=6.0 \mathrm{~m} \mathrm{~L} 2 / \mathrm{L} 1=0.5$

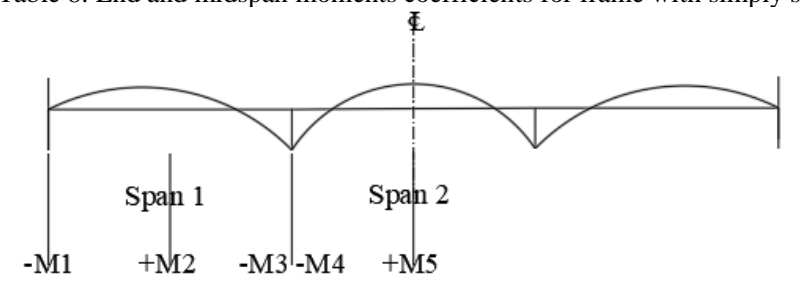

\begin{tabular}{|c|c|c|c|c|c|c|c|}
\hline & \multirow[t]{2}{*}{ Column Dimension (mm) } & \multirow[t]{2}{*}{ Beam Dimension (mm) } & \multicolumn{3}{|c|}{ Span 1} & \multicolumn{2}{|c|}{ Span 2} \\
\hline & & & $-\mathrm{M} 1$ & $+\mathrm{M} 2$ & -M3 & $-\mathrm{M} 4$ & $+\mathrm{M} 5$ \\
\hline DDM & Span 1: Case(a) & & 0 & 0.63 & 0.75 & 0.65 & 0.35 \\
\hline & Span 2: Case (e) & & & & & & \\
\hline EFM & $500 \times 500$ & $500 \times 700$ & 0 & 0.572 & 0.856 & 0.720 & 0.280 \\
\hline & $400 \times 400$ & $400 \times 600$ & 0 & 0.574 & 0.852 & 0.730 & 0.270 \\
\hline & $300 \times 300$ & $300 \times 500$ & 0 & 0.577 & 0.845 & 0.745 & 0.255 \\
\hline Average & & & 0 & 0.575 & 0.850 & 0.732 & 0.268 \\
\hline FEM & $500 \times 500$ & $500 \times 700$ & 0 & 0.571 & 0.858 & 0.715 & 0.285 \\
\hline & $400 \times 400$ & $400 \times 600$ & 0 & 0.572 & 0.856 & 0.727 & 0.273 \\
\hline & $300 \times 300$ & $300 \times 500$ & 0 & 0.576 & 0.848 & 0.741 & 0.259 \\
\hline Average & & & 0 & 0.573 & 0.854 & 0.728 & 0.272 \\
\hline
\end{tabular}

Table 9. End moment at face of support and midspan moments coefficients for frame $\mathrm{L} 1=6.0 \mathrm{~m}, \mathrm{~L} 2=6.0 \mathrm{~m} \mathrm{~L} 2 / \mathrm{L} 1=1.0$

\begin{tabular}{|c|c|c|c|c|c|c|c|c|}
\hline \multirow[t]{3}{*}{-M1 } & \multirow{3}{*}{$\begin{array}{r}\text { Span } 1 \\
+\mathrm{M} 2 \\
\mathrm{C}\end{array}$} & $\begin{array}{ll}\text { Span } 2 \\
-\mathrm{M} 3-\mathrm{M} 4 \quad+\mathrm{M} 5\end{array}$ & & & & & & \\
\hline & & \multirow[t]{2}{*}{ Column Dimension (mm) } & \multirow[t]{2}{*}{ Beam Dimension $(\mathrm{mm})$} & \multicolumn{3}{|c|}{ Span 1} & \multicolumn{2}{|c|}{ Span 2} \\
\hline & & & & $-\mathrm{M} 1$ & $+\mathrm{M} 2$ & $-\mathrm{M} 3$ & $-\mathrm{M} 4$ & $+\mathrm{M} 5$ \\
\hline DDM & & $\begin{array}{l}\text { an 1: Case(b) } \\
\text { an 2: Case (e) }\end{array}$ & & 0.16 & 0.57 & 0.70 & 0.65 & 0.35 \\
\hline EFM & & $0 \times 500$ & $\begin{array}{l}500 \times 700 \\
400 \times 600\end{array}$ & $\begin{array}{l}0.118 \\
0.111\end{array}$ & $\begin{array}{l}0.588 \\
0.575\end{array}$ & $\begin{array}{l}0.706 \\
0.738\end{array}$ & $\begin{array}{l}0.679 \\
0.698\end{array}$ & $\begin{array}{l}0.321 \\
0.302\end{array}$ \\
\hline Average & & $0 \times 300$ & $300 \times 500$ & $\begin{array}{l}0.073 \\
0.101\end{array}$ & $\begin{array}{l}0.637 \\
0.591\end{array}$ & $\begin{array}{l}0.707 \\
0.717\end{array}$ & $\begin{array}{l}0.708 \\
0.695\end{array}$ & $\begin{array}{l}0.292 \\
0.305\end{array}$ \\
\hline FEM & & $\begin{array}{l}0 \times 500 \\
0 \times 400\end{array}$ & $\begin{array}{l}500 \times 700 \\
400 \times 600\end{array}$ & $\begin{array}{l}0.269 \\
0.252\end{array}$ & $\begin{array}{l}0.522 \\
0.518\end{array}$ & $\begin{array}{l}0.687 \\
0.712\end{array}$ & $\begin{array}{l}0.640 \\
0.660\end{array}$ & $\begin{array}{l}0.360 \\
0.340\end{array}$ \\
\hline Average & & $0 \times 300$ & $300 \times 500$ & $\begin{array}{l}0.219 \\
0.247\end{array}$ & $\begin{array}{l}0.523 \\
0.520\end{array}$ & $\begin{array}{l}0.736 \\
0.712\end{array}$ & $\begin{array}{l}0.685 \\
0.662\end{array}$ & $\begin{array}{l}0.315 \\
0.338\end{array}$ \\
\hline
\end{tabular}


Table 10. End and midspan moments coefficients for frame with simply supported external support, $\mathrm{L} 1=6.0 \mathrm{~m}, \mathrm{~L} 2=6.0 \mathrm{~m} \mathrm{~L} 2 / \mathrm{L} 1=1.0$

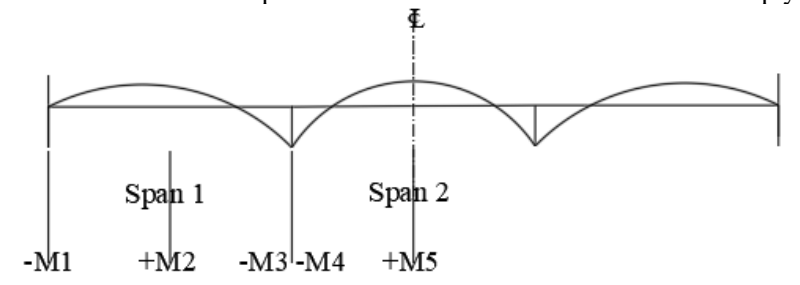

\begin{tabular}{|c|c|c|c|c|c|c|c|}
\hline & \multirow{2}{*}{ Column Dimension (mm) } & \multirow{2}{*}{ Beam Dimension (mm) } & \multicolumn{3}{|c|}{ Span 1} & \multicolumn{2}{|c|}{ Span 2} \\
\hline & & & -M1 & $+\mathrm{M} 2$ & -M3 & -M4 & $+\mathrm{M} 5$ \\
\hline \multirow[t]{2}{*}{ DDM } & Span 1: Case(a) & & 0 & 0.63 & 0.75 & 0.65 & 0.35 \\
\hline & Span 2: Case (e) & & & & & & \\
\hline \multirow[t]{3}{*}{ EFM } & $500 \times 500$ & $500 \times 700$ & 0 & 0.620 & 0.760 & 0.720 & 0.280 \\
\hline & $400 \times 400$ & $400 \times 600$ & 0 & 0.604 & 0.792 & 0.734 & 0.266 \\
\hline & $300 \times 300$ & $300 \times 500$ & 0 & 0.655 & 0.690 & 0.650 & 0.350 \\
\hline Average & & & 0 & 0.627 & 0.747 & 0.701 & 0.299 \\
\hline \multirow[t]{3}{*}{ FEM } & $500 \times 500$ & $500 \times 700$ & 0 & 0.617 & 0.766 & 0.699 & 0.301 \\
\hline & $400 \times 400$ & $400 \times 600$ & 0 & 0.610 & 0.780 & 0.722 & 0.278 \\
\hline & $300 \times 300$ & $300 \times 500$ & 0 & 0.626 & 0.748 & 0.703 & 0.297 \\
\hline Average & & & 0 & 0.618 & 0.765 & 0.708 & 0.292 \\
\hline
\end{tabular}

Table 11. End moment at face of support and midspan moments coefficients for frame $\mathrm{L} 1=3.0 \mathrm{~m}, \mathrm{~L} 2=6.0 \mathrm{~m} \mathrm{~L} 2 / \mathrm{L} 1=2.0$

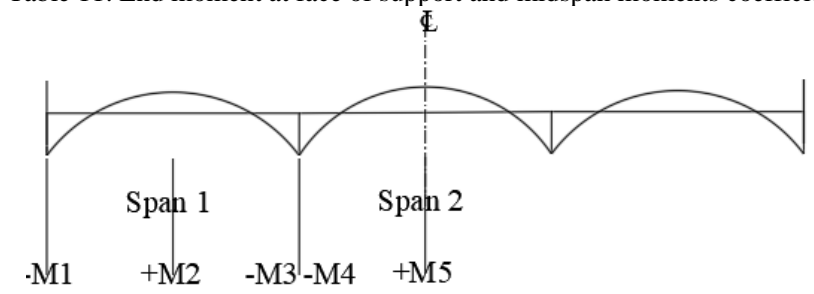

\begin{tabular}{|c|c|c|c|c|c|c|c|}
\hline & \multirow{2}{*}{ Column Dimension (mm) } & \multirow{2}{*}{ Beam Dimension (mm) } & \multicolumn{3}{|c|}{ Span 1} & \multicolumn{2}{|c|}{ Span 2} \\
\hline & & & -M1 & $+\mathrm{M} 2$ & -M3 & -M4 & +M5 \\
\hline \multirow[t]{2}{*}{ DDM } & Span 1: Case(b) & & 0.16 & 0.57 & 0.70 & 0.65 & 0.35 \\
\hline & Span 2: Case (e) & & & & & & \\
\hline \multirow[t]{3}{*}{ EFM } & $500 \times 500$ & $500 \times 700$ & +0.155 & 0.764 & 0.628 & 0.627 & 0.373 \\
\hline & $400 \times 400$ & $400 \times 600$ & +0.107 & 0.718 & 0.670 & 0.669 & 0.331 \\
\hline & $300 \times 300$ & $300 \times 500$ & +0.071 & 0.682 & 0.707 & 0.708 & 0.292 \\
\hline Average & & & +0.111 & 0.722 & 0.688 & 0.668 & 0.332 \\
\hline \multirow[t]{3}{*}{ FEM } & $500 \times 500$ & $500 \times 700$ & 0.100 & 0.710 & 0.480 & 0.524 & 0.476 \\
\hline & $400 \times 400$ & $400 \times 600$ & 0.076 & 0.672 & 0.580 & 0.584 & 0.416 \\
\hline & $300 \times 300$ & $300 \times 500$ & 0.065 & 0.646 & 0.645 & 0.640 & 0.360 \\
\hline Average & & & 0.080 & 0.676 & 0.568 & 0.583 & 0.417 \\
\hline
\end{tabular}

Table 12. End and midspan moments coefficients for frame with simply supported external support, $\mathrm{L} 1=3.0 \mathrm{~m}, \mathrm{~L} 2=6.0 \mathrm{~m} \mathrm{~L} 2 / \mathrm{L} 1=2.0$

\begin{tabular}{|c|c|c|c|c|c|c|c|}
\hline & \multirow[t]{2}{*}{ Column Dimension (mm) } & \multirow[t]{2}{*}{ Beam Dimension (mm) } & \multicolumn{3}{|c|}{ Span 1} & \multicolumn{2}{|c|}{ Span 2} \\
\hline & & & $-\mathrm{M} 1$ & $+\mathrm{M} 2$ & -M3 & -M4 & +M5 \\
\hline \multirow[t]{2}{*}{ DDM } & Span 1: Case(a) & & 0 & 0.63 & 0.75 & 0.65 & 0.35 \\
\hline & Span 2: Case (e) & & & & & & \\
\hline \multirow[t]{3}{*}{ EFM } & $500 \times 500$ & $500 \times 700$ & 0 & 0.671 & 0.657 & 0.661 & 0.339 \\
\hline & $400 \times 400$ & $400 \times 600$ & 0 & 0.652 & 0.696 & 0.697 & 0.303 \\
\hline & $300 \times 300$ & $300 \times 500$ & 0 & 0.636 & 0.728 & 0.730 & 0.270 \\
\hline Average & & & 0 & 0.653 & 0.694 & 0.696 & 0.304 \\
\hline \multirow[t]{3}{*}{ FEM } & $500 \times 500$ & $500 \times 700$ & 0 & 0.752 & 0.497 & 0.540 & 0.460 \\
\hline & $400 \times 400$ & $400 \times 600$ & 0 & 0.710 & 0.580 & 0.607 & 0.393 \\
\hline & $300 \times 300$ & $300 \times 500$ & 0 & 0.676 & 0.648 & 0.663 & 0.337 \\
\hline Average & & & 0 & 0.713 & 0.575 & 0.603 & 0.397 \\
\hline
\end{tabular}

Table 13. Fixed End moment at face of support and midspan moments coefficients for Flat plate frame system (3, 4 \& 5) equal spans. Results of problem 7,8 \&9

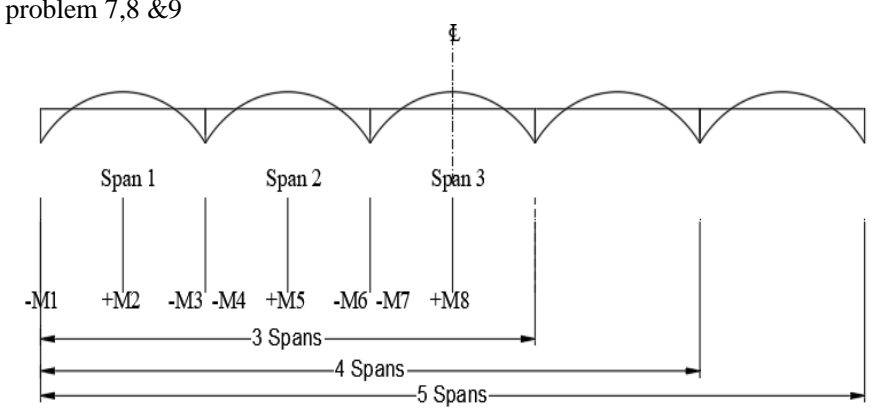




\begin{tabular}{|c|c|c|c|c|c|c|c|c|}
\hline \multirow[b]{3}{*}{ Without edge beam } & \multicolumn{3}{|c|}{ Span 1} & & \multicolumn{2}{|c|}{ Span 2} & \multicolumn{2}{|c|}{ Span 3} \\
\hline & \multicolumn{4}{|c|}{3 Spans } & & \multirow[b]{2}{*}{-M6 } & \multirow[b]{2}{*}{-M7 } & \multirow[b]{2}{*}{$+\mathrm{M} 8$} \\
\hline & -M1 & $+\mathrm{M} 2$ & -M3 & -M4 & +M5 & & & \\
\hline DDM: Case c, e & 0.26 & 0.52 & 0.70 & 0.65 & 0.35 & & & \\
\hline EFM & 0.260 & 0.517 & 0.706 & 0.643 & 0.357 & & & \\
\hline \multicolumn{9}{|l|}{ FEM } \\
\hline With edge beam & -M1 & $+\mathrm{M} 2$ & -M3 & $-\mathrm{M} 4$ & +M5 & -M6 & -M7 & +M8 \\
\hline DDM: Case d, e & 0.30 & 0.50 & 0.70 & 0.65 & 0.35 & & & \\
\hline EFM & 0.440 & 0.447 & 0.666 & 0.613 & 0.387 & & & \\
\hline \multicolumn{9}{|c|}{4 Spans } \\
\hline Without edge beam & -M1 & $+\mathrm{M} 2$ & -M3 & $-\mathrm{M} 4$ & +M5 & -M6 & $-\mathrm{M} 7$ & +M8 \\
\hline DDM: Case c, e & 0.26 & 0.52 & 0.70 & 0.65 & 0.35 & 0.65 & & \\
\hline EFM & 0.256 & 0.514 & 0.717 & 0.665 & 0.380 & 0.575 & & \\
\hline With edge beam & -M1 & $+\mathrm{M} 2$ & -M3 & -M4 & +M5 & -M6 & -M7 & +M8 \\
\hline DDM: Case d, e & 0.30 & 0.50 & 0.70 & 0.65 & 0.35 & 0.65 & & \\
\hline EFM & 0.439 & 0.447 & 0.667 & 0.619 & 0.393 & 0.596 & & \\
\hline \multicolumn{9}{|c|}{5 Spans } \\
\hline Without edge beam & -M1 & $+\mathrm{M} 2$ & -M3 & $-\mathrm{M} 4$ & +M5 & -M6 & -M7 & +M8 \\
\hline DDM: Case c, e & 0.26 & 0.52 & 0.70 & 0.65 & 0.35 & 0.65 & 0.65 & 0.35 \\
\hline EFM & 0.257 & 0.514 & 0.715 & 0.661 & 0.377 & 0.586 & 0.597 & 0.403 \\
\hline \multicolumn{9}{|l|}{ FEM } \\
\hline With edge beam & -M1 & $+\mathrm{M} 2$ & -M3 & -M4 & +M5 & -M6 & $-\mathrm{M} 7$ & +M8 \\
\hline DDM: Case d, e & 0.30 & 0.50 & 0.70 & 0.65 & 0.35 & 0.65 & 0.65 & 0.35 \\
\hline EFM & 0.439 & 0.447 & 0.667 & 0.619 & 0.392 & 0.597 & 0.602 & 0.398 \\
\hline
\end{tabular}

*The FEM analysis doesn't apply because the results of the DDM \& EFM are close to each other.

Table 14. Fixed end moment at face of support and midspan moments coefficients for column-beam frame and Flat plate system three different length spans, the middle span is less than outer span by $33 \%$. Results of problem 10

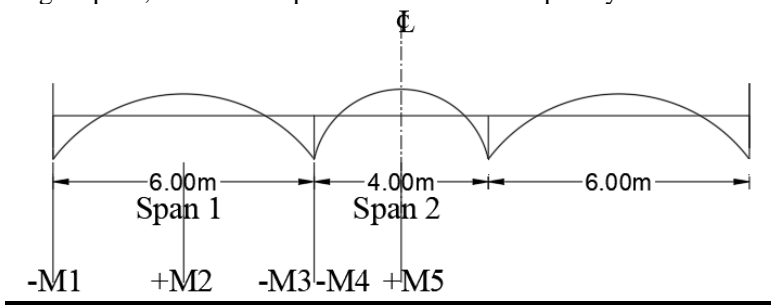

\begin{tabular}{llllll}
\hline & \multicolumn{3}{c}{ Span 1: 6.0m } & \multicolumn{2}{c}{ Span 2 } \\
Beam-column system & $-\mathrm{M} 1$ & $+\mathrm{M} 2$ & $-\mathrm{M} 3$ & $-\mathrm{M} 4$ & 0.35 \\
\hline DDM: span 1: case b & 0.16 & 0.57 & 0.70 & 0.65 & -0.205 \\
span 2: case e & & & & & \\
EFM & 0.139 & 0.648 & 0.566 & 1.205 & 0 \\
FEM & 0.294 & 0.572 & 0.562 & 1.000 & $+\mathrm{M} 5$ \\
With edge beam & $-\mathrm{M} 1$ & $+\mathrm{M} 2$ & $-\mathrm{M} 3$ & $-\mathrm{M} 4$ & 0.35 \\
DDM: Case d, e & 0.30 & 0.50 & 0.70 & 0.65 & 0.247 \\
EFM & 0.292 & 0.566 & 0.576 & 1.000 & 0.753 \\
FEM & 0.475 & 0.470 & 0.586 & & 0.25 \\
\hline
\end{tabular}

Table 15. Fixed end moment at face of support and midspan moments coefficients for column-beam frame and Flat plate system three different length spans, the external or outer support is simply supported. Results of problem 11

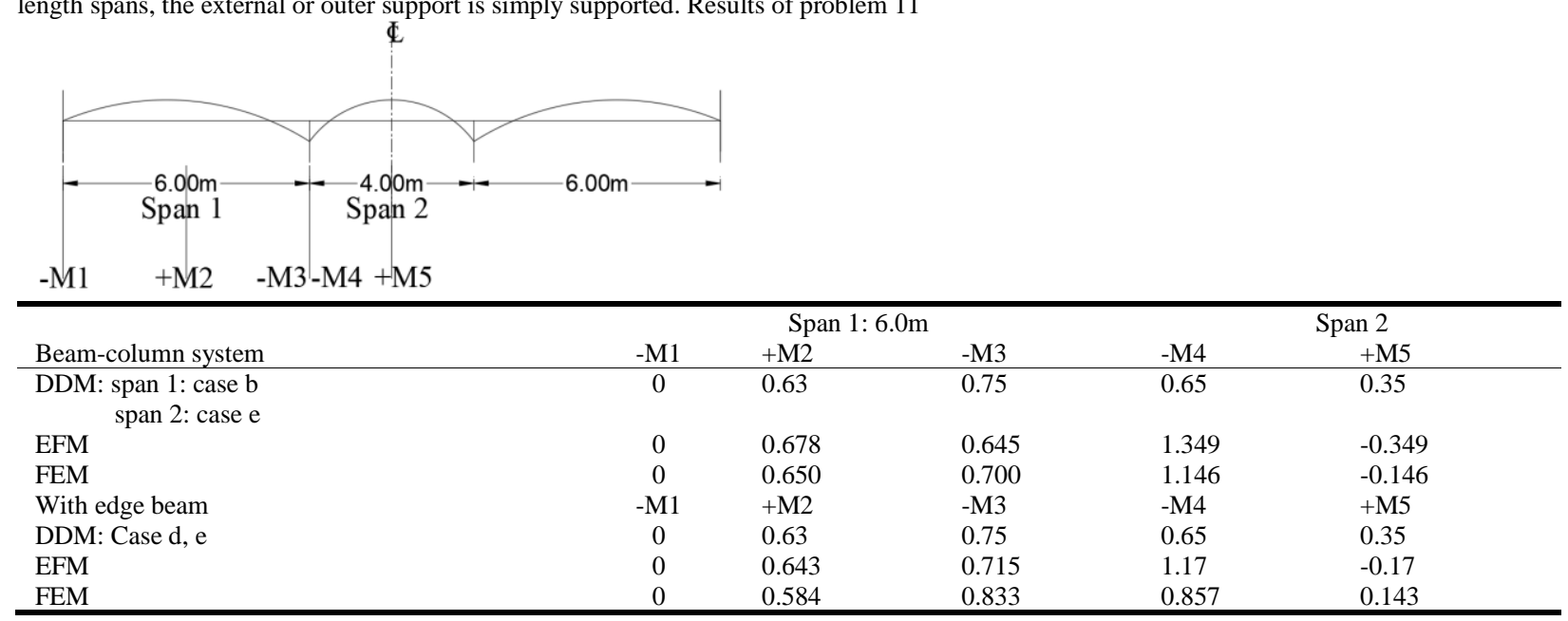


Table 16. Fixed end and midspan moments coefficients for three spans different spans, the middle span is greater than outer by (33\%). Results of problem 12.

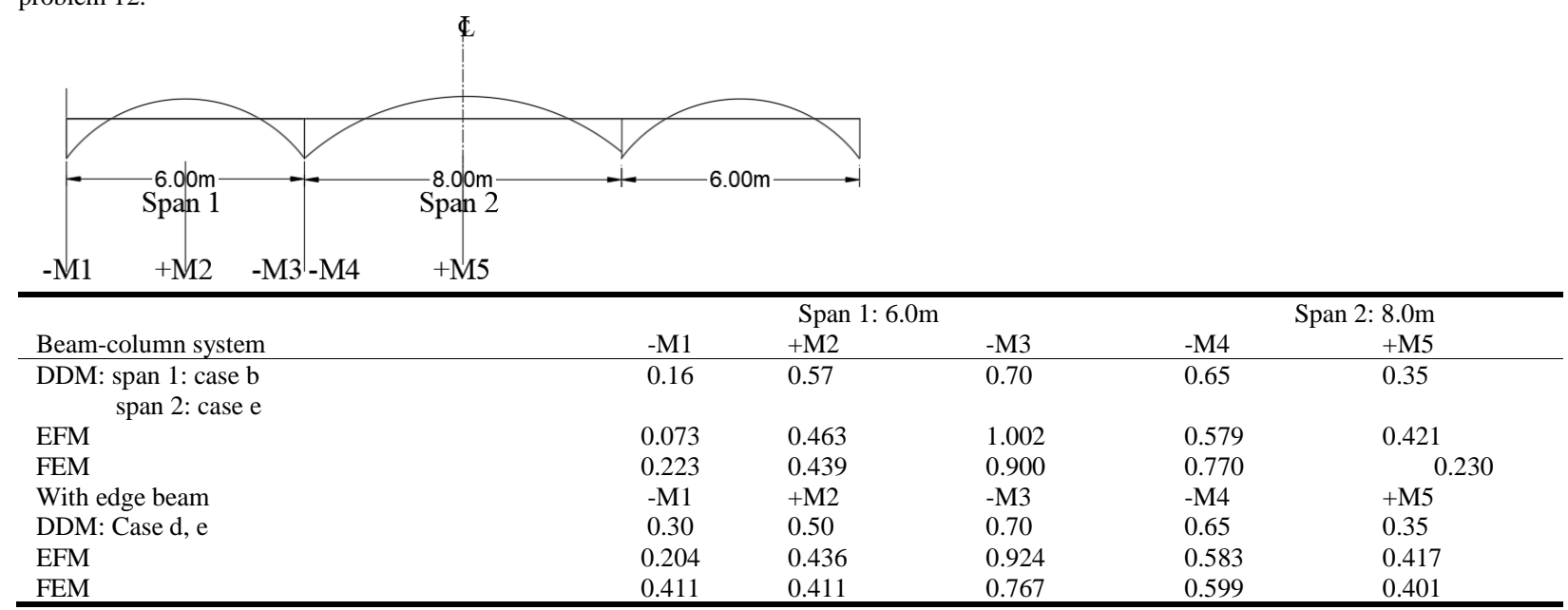

Table 17. Fixed end and midspan moments coefficients for three spans different spans, the external support is simply supported. Results of problem 13

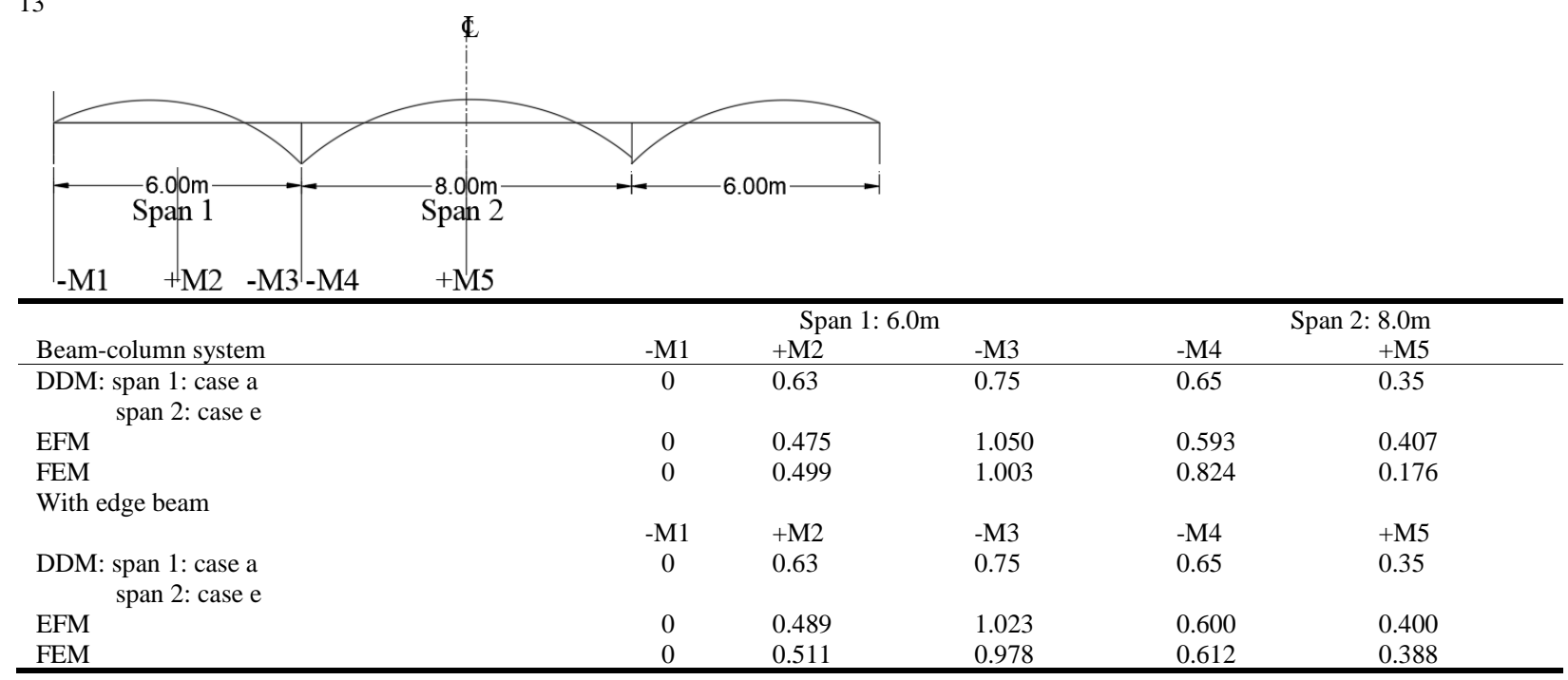

Table 18. End moment at face of support and midspan moments coefficients for four equal span frame $\mathrm{L} 1=6.0 \mathrm{~m}, \mathrm{~L} 2=6.0 \mathrm{~m} \mathrm{~L} 2 / \mathrm{L} 1=1.0$. Results of problem 14

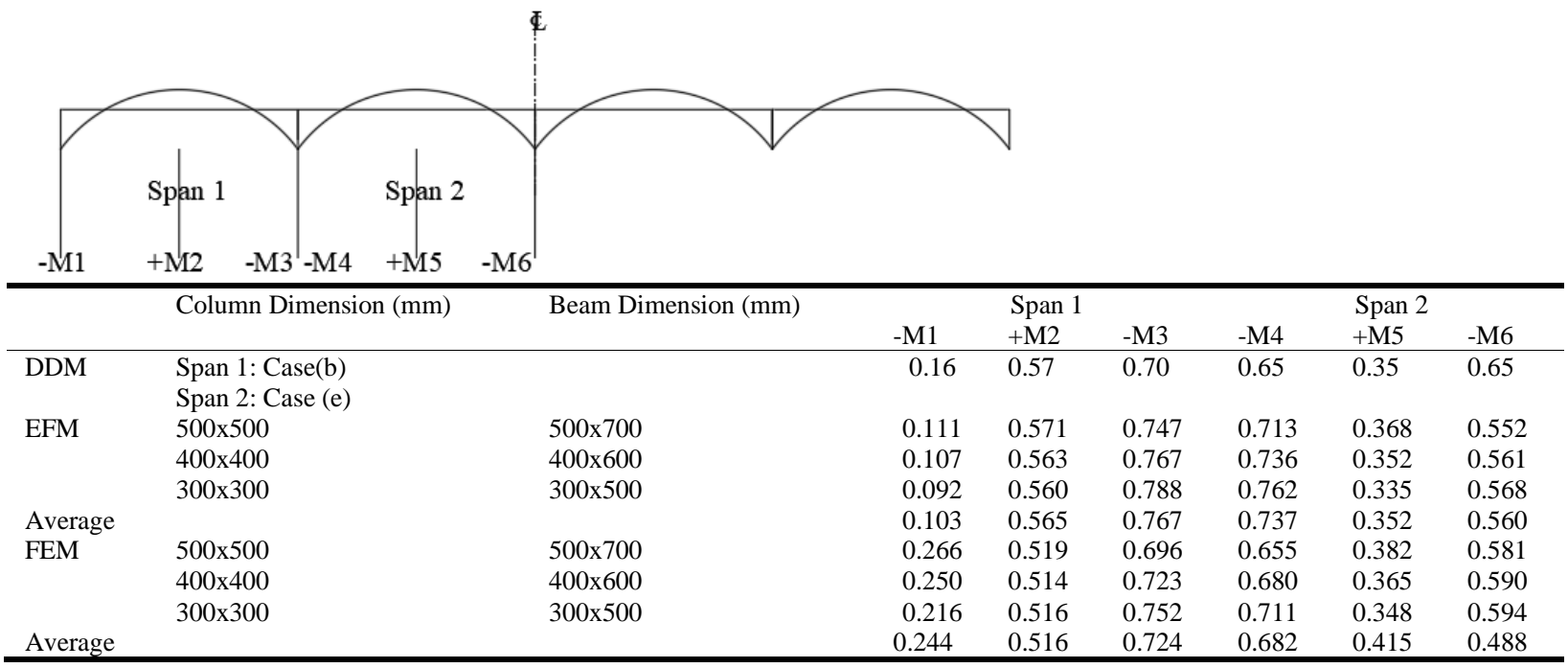


Table 19. End moment at face of support and midspan moments coefficients for four equal span frame $\mathrm{L} 1=6.0 \mathrm{~m}, \mathrm{~L} 2=6.0 \mathrm{~m} \mathrm{~L} / \mathrm{L} 1=1.0$ the external or outer support is simply supported. Results of problem 15

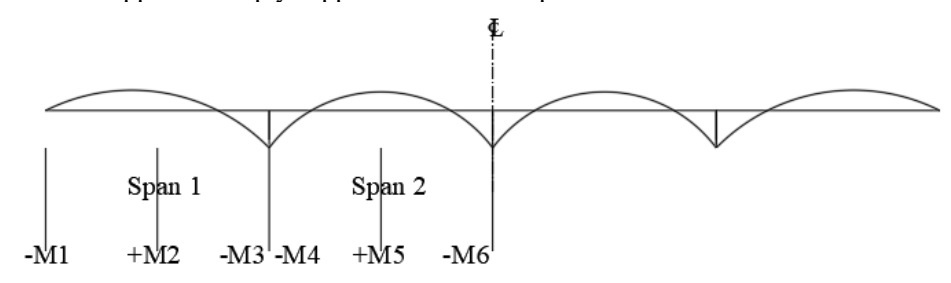

\begin{tabular}{|c|c|c|c|c|c|c|c|c|}
\hline & \multirow[t]{2}{*}{ Column Dimension (mm) } & \multirow[t]{2}{*}{ Beam Dimension (mm) } & \multicolumn{3}{|c|}{ Span 1} & \multicolumn{3}{|c|}{ Span 2} \\
\hline & & & -M1 & $+\mathrm{M} 2$ & -M3 & $-\mathrm{M} 4$ & $+\mathrm{M} 5$ & -M6 \\
\hline \multirow[t]{2}{*}{ DDM } & Span 1: Case(b) & & 0 & 0.63 & 0.75 & 0.65 & 0.35 & 0.65 \\
\hline & Span 2: Case (e) & & & & & & & \\
\hline \multirow[t]{3}{*}{ EFM } & $500 \times 500$ & $500 \times 700$ & 0 & 0.589 & 0.822 & 0.770 & 0.352 & 0.526 \\
\hline & $400 \times 400$ & $400 \times 600$ & 0 & 0.584 & 0.832 & 0.787 & 0338 & 0.538 \\
\hline & $300 \times 300$ & $300 \times 500$ & 0 & 0.580 & 0.840 & $0 . .805$ & 0.324 & 0.547 \\
\hline Average & & & 0 & 0.585 & 0.831 & 0.787 & 0.338 & 0.537 \\
\hline \multirow[t]{3}{*}{ FEM } & $500 \times 500$ & $500 \times 700$ & 0 & 0.604 & 0.792 & 0.741 & 0.357 & 0.546 \\
\hline & $400 \times 400$ & $400 \times 600$ & 0 & 0.595 & 0.810 & 0.764 & 0.341 & 0.554 \\
\hline & $300 \times 300$ & $300 \times 500$ & 0 & 0.588 & 0.824 & 0.787 & 0.327 & 0.560 \\
\hline Average & & & 0 & 0.596 & 0.809 & 0.764 & 0.342 & 0.553 \\
\hline
\end{tabular}

Table 2.0 End and midspan moments coefficients for five equal span frame $\mathrm{L} 1=6.0 \mathrm{~m}, \mathrm{~L} 2=6.0 \mathrm{~m} \mathrm{~L} 2 / \mathrm{L} 1=1.0$. Results of problem 16

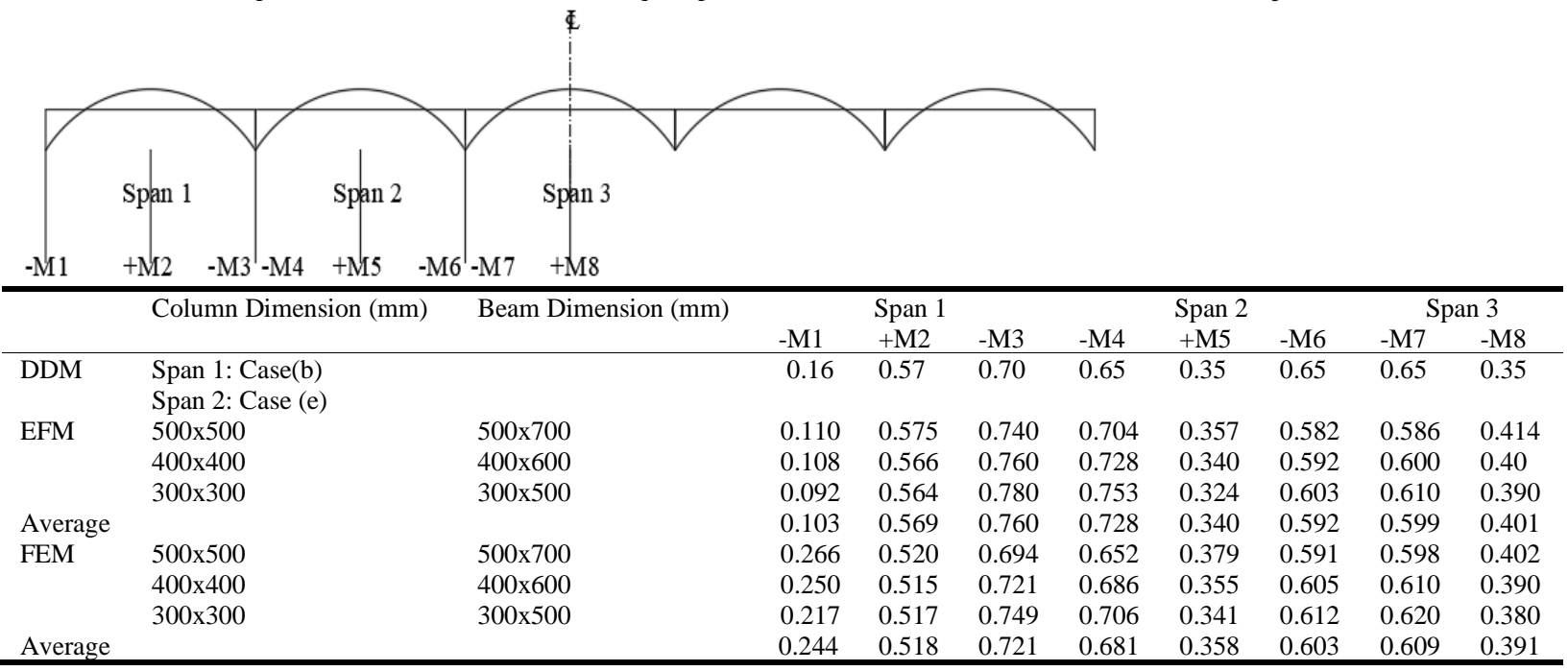

Table 21. End and midspan moments coefficients for five equal span frames with external simple support, $\mathrm{L} 1=6.0 \mathrm{~m}, \mathrm{~L} 2=6.0 \mathrm{~m} \mathrm{~L} 2 / \mathrm{L} 1=1.0$. Results of problem 17

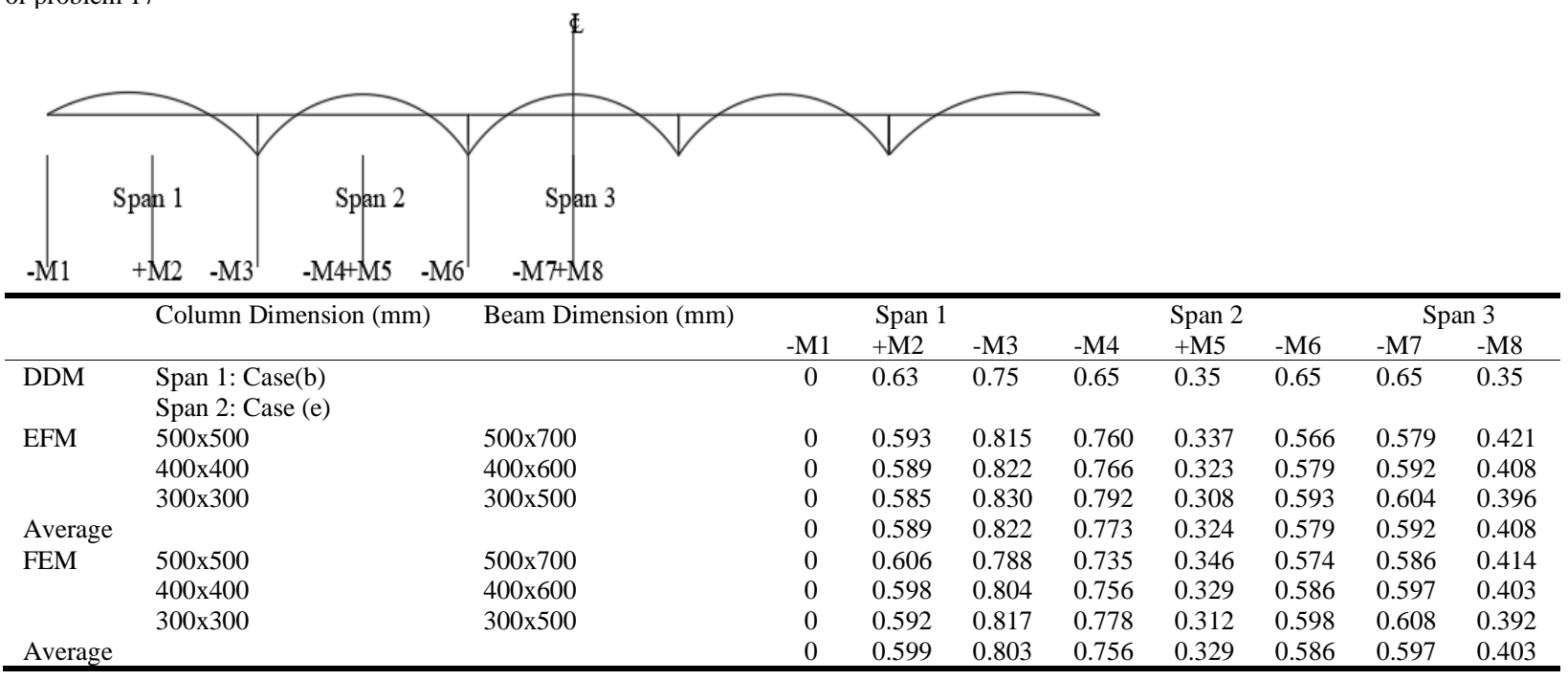




\section{RESULTS AND DISCUSSION}

Table (1) \& Fig (1), show that (-ve) end moments at the face of external support and (-ve) moment at internal support of 1 st outer span decreased with increasing the stiffness ratio $(\boldsymbol{\psi})$ or panel ratio (L2/L1), while the mid span (+ve) moment increased with increasing the stiffness ratio $(\boldsymbol{\psi})$ or panel ratio (L2/L1).

The same behavior is noticed when the beam and column size is changed as shown in fig. $2 \& 3)$ and table $(3 \& 5)$. In the middle span, the end (-ve) moment at interior support increased with increasing $(\boldsymbol{\psi})$ or (L2/L1), while (+ve) midspan moment decreased with increasing $(\boldsymbol{\psi})$ or $(\mathrm{L} 2 / \mathrm{L} 1)$. In the middle span, the (-ve) moment at internal support increased with increasing stiffness ratio $(\boldsymbol{\psi})$ or panel ratio (L2/L1), while (+ve) midspan moment decreased.

When the outer support is simply supported, the (-ve) end moment at the internal support the first span decreased with increasing the stiffness ratio or panel ratio $(\mathrm{L} 2 / \mathrm{L} 1)$ while the midspan (+ve) moment at first span increased with increasing $(\boldsymbol{\psi}$ or L2/L1) as shown in table (2). Same behavior is noticed when the column $\&$ beam sizes are changed as shown in table
(4 \& 6). Tables ( 7 to 12 ) show the end and midspan moment coefficient comparison between three methods (DDM, EFM \& EFM) for different column and beam sizes and panel ratio (L2/L1), the results of EFM \& FEM is differ than DDM, so (EFM and FEM) is recommended to analyze the frames. Also, the changing of column and beam sizes have small effects on the moment coefficients.

The external (-ve) end moment of the 1st span deceased with decreasing the column and beam sizes, while the (-ve) end moment at the internal support and midspan (+ve) moments are increased, for the 2 nd span, the (-ve) end moment at internal support increased with increasing the column \& beam sizes, while the midspan (+ve) moment is decreased. The same conclusions are noticed for all panel ratios (L2/L1=0.5, $1 \& 2)$ as shown in table $(7,9 \& 11)$.

For frames with external support is simply supported, the results obtained from (EFM and FEM) are very close to each other and approximately reached to DDM results for $(\mathrm{L} 2 / \mathrm{L} 1=0.5,1 \& 2)$ as shown in tables $(8,10 \& 12)$.

From the previous tables (7 to 12); the new moment coefficients are suggested to modify the DDM moment coefficients as shown in the following tables (22):

Table 22. The suggested envelope for 3 spans

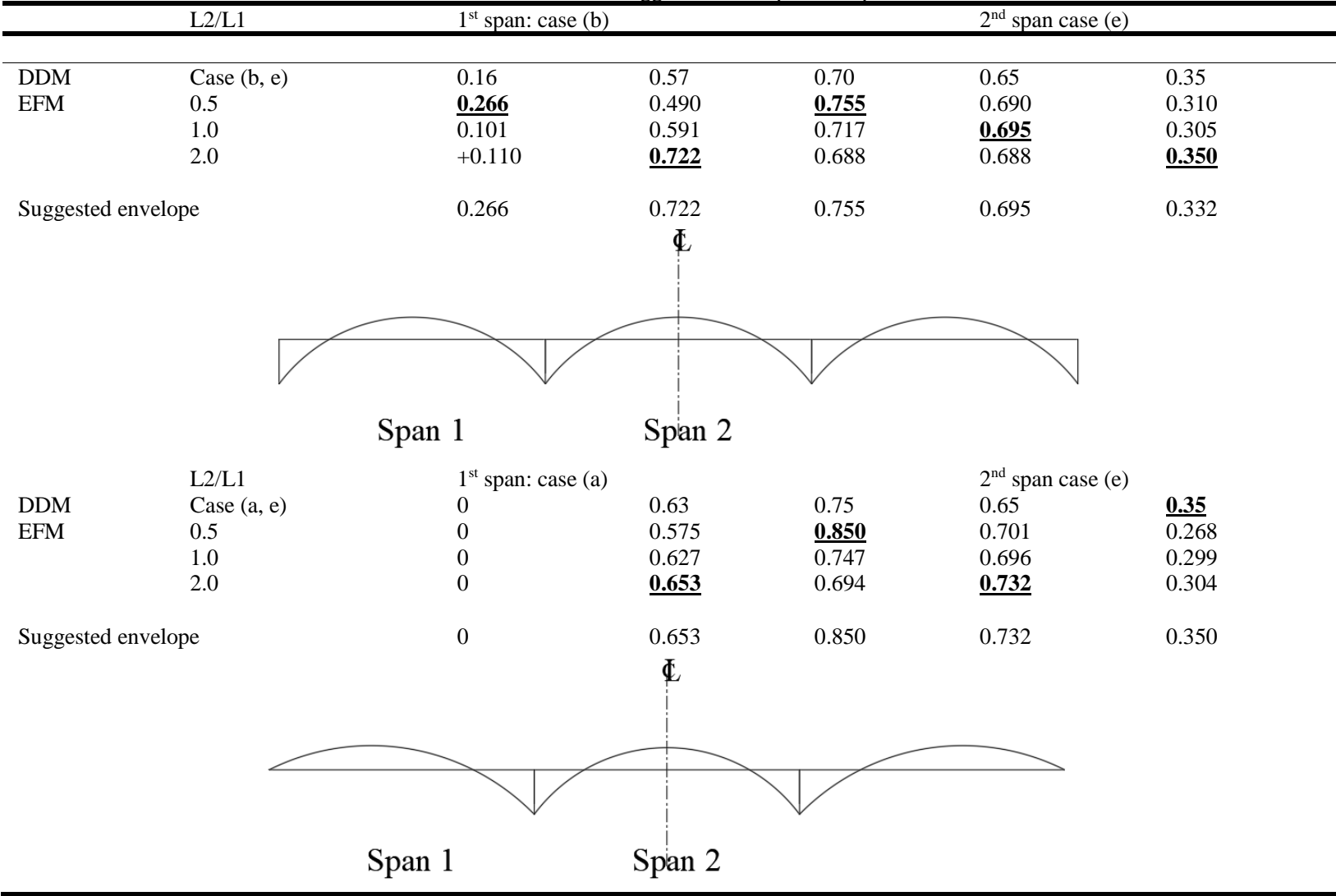

The suggested envelope based on the result of EFM, the FEM can be used in all cases because its more accurate and more reliable and computer software are used.

Table (13); shows the moment coefficient of the end moment and midspan moment for flat plate slab system for 3, 4 and 5 equal spans, $\mathrm{L} 2 / \mathrm{L} 1=1.0$, as shown the result of $\mathrm{EFM}$ are close to the DDM results in case of flat plate slab system without edge beam. Thus, the DDM moment coefficient (case c) recommended for the analysis and design.

In case the flat plate slab system with edge beam, the results between DDM \& EFM are differ, so EFM is recommended for analysis and determining moments.

supported. The results of the three methods and comparison are shown in tables (14 to 17).
The (-ve) end moment in EFM at the external support 1st span increased, while the (-ve) moments at all other supports are decreased compared with DDM. The (+ve) midspan moment of 1st span decreased in (EFM), while midspan of 2nd span is increased compared with DDM.

When the middle span differs than outer span by $( \pm 33 \%)$. The result of the two methods (EFM \& FEM) are quite differ than DDM, so using of EFM or FEM are recommended for the analysis and design because in case of the 2nd span when less than 1 st outer span by $(-33 \%)$, negative moment is occurred at midspan, which is opposite to the results of DDM. The same behavior is obtained when the outer external support is simply Tables (18 to 21) show the moment coefficient for 4 equal spans and 5 spans frame system $(\mathrm{L} 2 / \mathrm{L} 1=1.0)$ for different column \& 
beam sizes and comparison between the three methods (DDM, EFM \& FEM), the same conclusions are obtained as in three equal span frames.

The results of EFM \& FEM are differed than DDM for all spans for four and five span frames, while the results of the three methods are close to each other in frames with external support is simply supported as shown in tables $(19 \& 21)$.
On the base of EFM; the moment coefficient of the DDM can be changed to the following suggested envelop as shown in the following table ( $23 \& 24)$. In all cases (FEM) can be adopted because its more accurate and convenient for computer use than other methods.

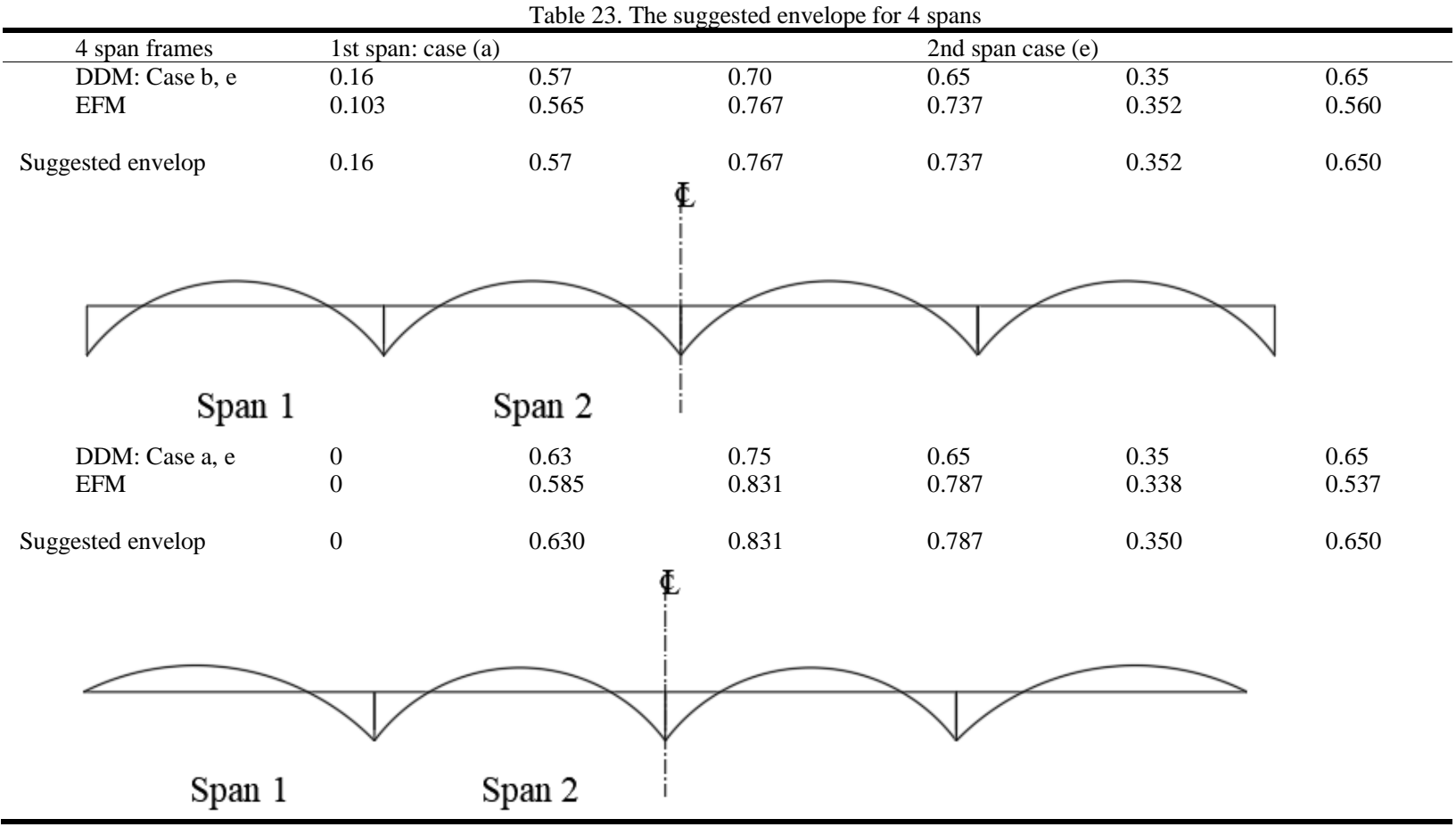

Table 24. The suggested envelope for 5 spans

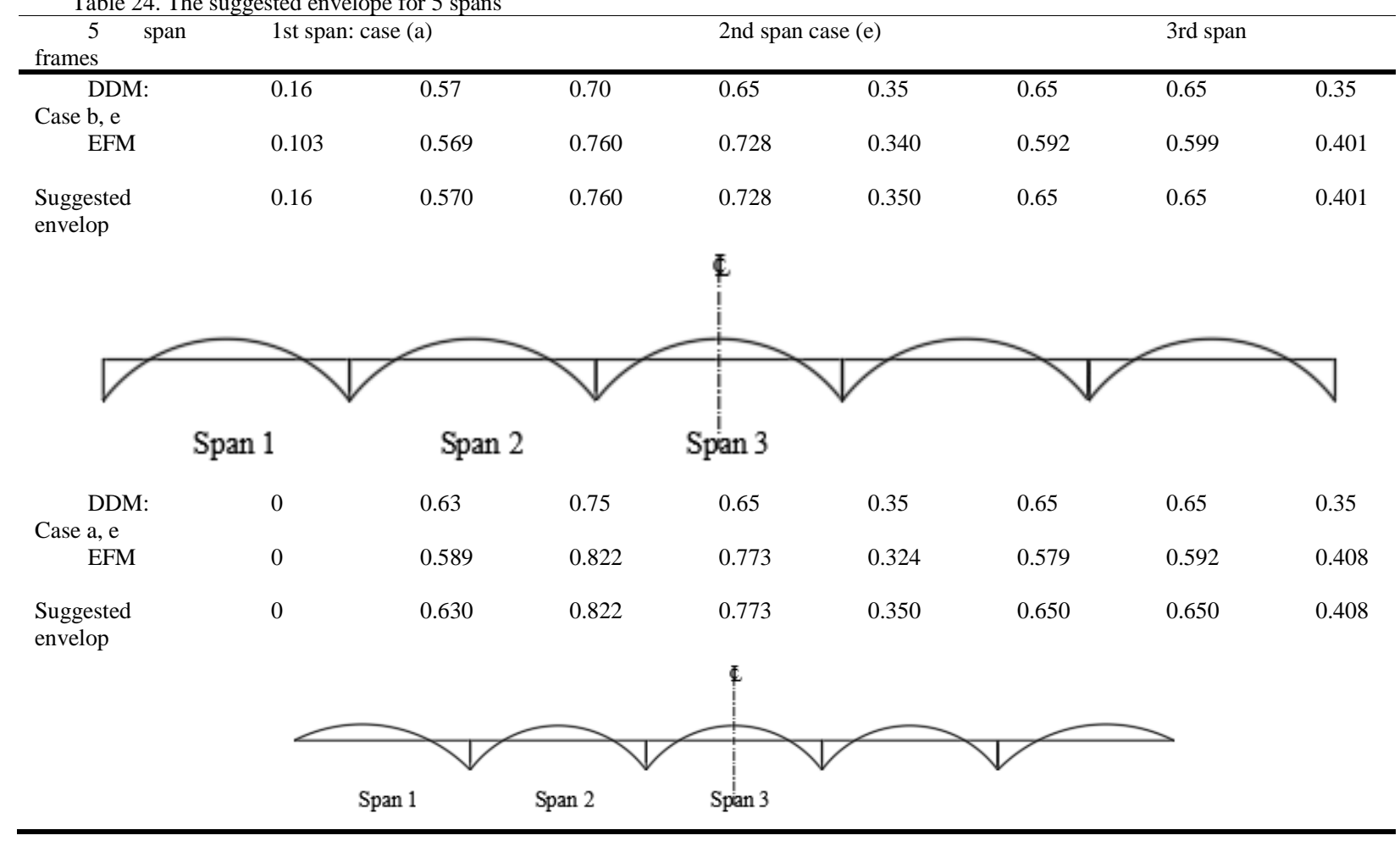




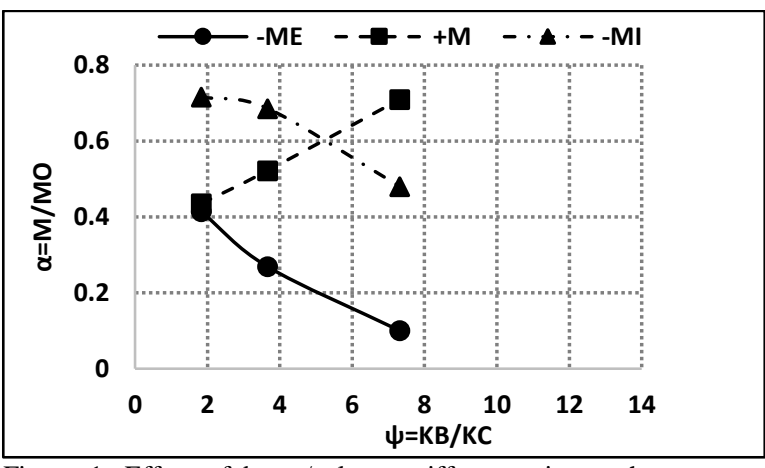

Figure 1. Effect of beam/column stiffness ratio on the moment Coefficient Column (500x500); beam (500x700)

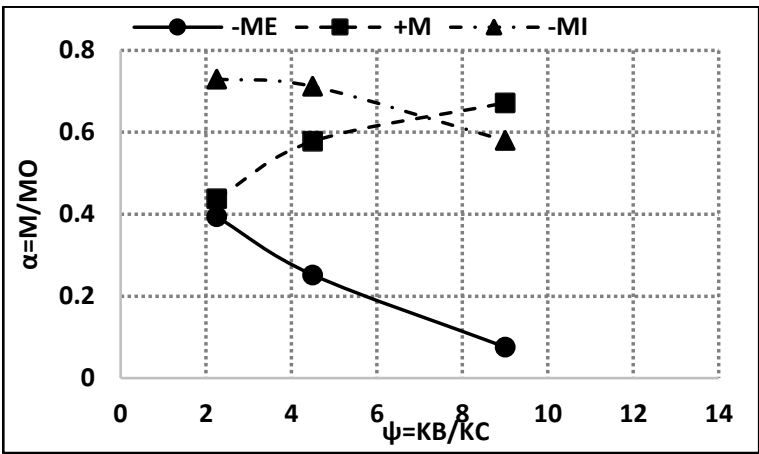

Figure 2. Effect of beam/column stiffness ratio on the moment Coefficient Column (400x400); beam (400x600)

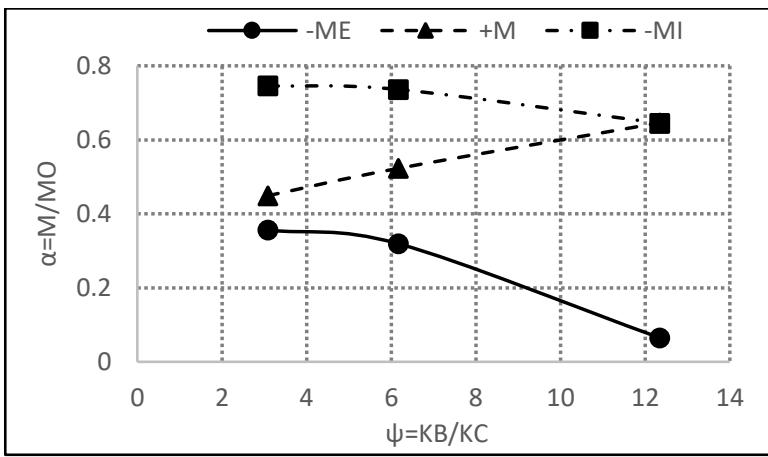

Figure 3. Effect of beam/column stiffness ratio on the moment Coefficient Column (300x300); beam (300x600)

\section{CONCLUSIONS AND RECOMMENDATIONS}

\subsection{Conclusions:}

The conclusions drawn from the current study could be summarized as below:

1- The (DDM) has some restrictions, hence (EFM) is adopted.

2- Both (DDM)\& (EFM) are approximate, butt results of (EFM) is more accurate

3- The (EFM) is not satisfactory for hand calculations, therefore use of computer software which based on FEM is adopted.

4- The results of EFM are more accurate as FEM takes into account the stiffness of the members, while DDM the coefficients of the moments are constant.

5- The -ve and +ve moments in EFM are calculated from the analysis of the frame, while in DDM the moments are constant and the total moment (Mo) distributed longitudinally by using moment coefficient cases $(\mathrm{a}, \mathrm{b}$, $\mathrm{c}, \mathrm{d}$ and $\mathrm{e})$.

6- The changing of column \& beam dimensions effect on the value of the moments, while in DDM is not changed.
7- Increasing the stiffness ratio $(\psi)$ or panel ratio (L2/L1) leads to decrease the (-ve) end moments at the face of external support and (-ve) moment at internal support of 1st outer span, while the mid span (+ve) moment increased with increasing the stiffness ratio

8- New moment coefficient envelopes are suggested instead of (DDM) coefficients as follows:

i. $\quad$ case (b, e): Three spans, and L2/L1 ranged from (0.5 to 2.0$)$

\begin{tabular}{llllll}
\hline Three & & Span1 & \multicolumn{3}{c}{ Span2 } \\
Span & $-\mathrm{M} 1$ & $+\mathrm{M} 2$ & $-\mathrm{M} 3$ & $-\mathrm{M} 4$ & $+\mathrm{M} 5$ \\
\hline $\begin{array}{l}\text { Suggested } \\
\text { envelope }\end{array}$ & 0.266 & 0.722 & 0.755 & 0.695 & 0.332 \\
\hline
\end{tabular}

ii. case (a, e): Three spans, and $\mathbf{L 2 / L 1}$ ranged from (0.5 to 2.0$)$

\begin{tabular}{llllll}
\hline Three & & Span1 & \multicolumn{3}{c}{ Span2 } \\
Span & $-\mathrm{M} 1$ & $+\mathrm{M} 2$ & $-\mathrm{M} 3$ & $-\mathrm{M} 4$ & $+\mathrm{M} 5$ \\
\hline $\begin{array}{l}\text { Suggested } \\
\text { envelope }\end{array}$ & 0 & 0.653 & 0.850 & 0.732 & 0.350 \\
\hline
\end{tabular}

iii. case (b, e): Four spans, and $\mathbf{L 2 / L 1}$ ranged from $(0.5$ to 2.0$)$

\begin{tabular}{lllllll}
\hline Four Span & \multicolumn{3}{c}{ Span1 } & \multicolumn{3}{c}{ Span2 } \\
& $-\mathrm{M} 1$ & $+\mathrm{M} 2$ & $-\mathrm{M} 3$ & $-\mathrm{M} 4$ & $+\mathrm{M} 5$ & $-\mathrm{M} 6$ \\
\hline $\begin{array}{l}\text { Suggested } \\
\text { envelope }\end{array}$ & 0.160 & 0.570 & 0.676 & 0.737 & 0.352 & 0.650 \\
\hline
\end{tabular}

iv. $\quad$ case (a, e): Four spans, and $\mathbf{L} 2 / \mathbf{L} 1$ ranged from ( 0.5 to 2.0 )

\begin{tabular}{|c|c|c|c|c|c|c|c|c|}
\hline \multirow{2}{*}{\multicolumn{2}{|c|}{ Four Span }} & \multicolumn{3}{|c|}{ Span1 } & \multicolumn{4}{|c|}{ Span2 } \\
\hline & & $\overline{\mathrm{M} 1}$ & $+\mathrm{M} 2$ & -M3 & -M4 & & & M6 \\
\hline \multicolumn{2}{|c|}{$\begin{array}{l}\text { Suggested } \\
\text { envelope }\end{array}$} & 0 & 0.630 & 0.831 & 0.787 & 0.35 & & .650 \\
\hline \multicolumn{2}{|c|}{ v. } & \multicolumn{7}{|c|}{$\begin{array}{l}\text { case (b, e): Five spans, and } \mathbf{L} 2 / \mathbf{L} \mathbf{1} \text { ranged from }(0.5 \\
\text { to } 2.0)\end{array}$} \\
\hline \multirow[t]{2}{*}{$\begin{array}{l}\text { Five } \\
\text { Span }\end{array}$} & & $\overline{\text { Spa }}$ & & & Span2 & & & \\
\hline & - & $\begin{array}{l}+\mathrm{M} \\
2\end{array}$ & -M3 & -M4 & $\begin{array}{l}+\mathrm{M} \\
5\end{array}$ & - & - & $\begin{array}{l}+\mathrm{M} \\
8\end{array}$ \\
\hline Sugges & 0.1 & 0.5 & 0.7 & 0.7 & 0.3 & 0.6 & 0.6 & 0.4 \\
\hline $\begin{array}{l}\text { ted } \\
\text { envelo } \\
\text { pe }\end{array}$ & 6 & 70 & 60 & 28 & 50 & 5 & 5 & 01 \\
\hline
\end{tabular}

vi. $\quad$ case (a, e): Five spans, and L2/L1 ranged from ( 0.5 to 2.0 )

\begin{tabular}{lllllllll}
\hline Five & \multicolumn{3}{c}{ Span1 } & \multicolumn{5}{c}{ Span2 } \\
Span & & & & & & & \\
& - & $+\mathrm{M}$ & $-\mathrm{M} 3$ & $-\mathrm{M} 4$ & $+\mathrm{M}$ & $-\mathrm{M} 6$ & $-\mathrm{M} 7$ & $+\mathrm{M}$ \\
& $\mathrm{M}$ & 2 & & & 5 & & & 8 \\
& 1 & & & & & & & \\
\hline $\begin{array}{l}\text { Sugges } \\
\text { ted }\end{array}$ & 0 & 0.6 & 0.8 & 0.7 & 0.3 & 0.6 & 0.6 & 0.4 \\
envelo & & 30 & 22 & 73 & 50 & 50 & 50 & 08 \\
pe & & & & & & & & \\
\hline
\end{tabular}

\subsection{Recommendations:}

1- EFM is more reliable method, because the moments are determined by stiffness and moment distribution method.

2- EFM is more accurate method and the moments are changing with changing the column, beam \& slab dimensions, while (DDM) the moment coefficients are constant.

3- EFM isn't satisfactory for hand calculations but can be computerized using computer software to solve the moment distribution with less time and errors. 
4- Computer software based on (FEM) can be used instead of (EFM).

\section{REFERENCES}

ACI Code (1963); "Building Code Requirements for Reinforced Concrete", ACI Publication 318-63, American Concrete Institute, Forming Hills, MI, 1963, USA.

ACI Code (2014). Building Code Requirements for Structural Concrete and Commentary, ACI 318-2014, American Concrete Institute, Forming Hills, MI 48331, USA, 520pp.

Darwin D., Dolon C. W. and Nilson A. H. (2016). Design of Concrete Structures, McoGrow Hill Education (India) Private Limited, Chennai, 15th edition, 780pp.

Patel J. J.and Dubey N. (2016). Comparison of Analysis Methods of Flat Slab, International Journal or Innovative
Research in Science \& Technology (IJIRST), No.2, Issue 12, May, pp. 459-463.

Patel S. S. and Sigi R. (2014). Analysis and Design of Flat Plat Slabs Using Various Codes, International Journal of Research in Science \& Technology (IJReT), Vo.3, Issue 4, April, pp. 417-421.

Hassan M. I. and Ahmed A. (2016). Design Comparison of Flat Plat System by Direct Design Method and Equivalent Frame Method,

https://www.researchgat.net/publication/281837415, 17 January.

Kudama L. (2015). Comparison of Equivalent Frame Analysis Results With Finite Element Analysis Results for Flat Plate Slab System, MSc thesis, Addis Ababa University, Addis Ababa Institute of Technology School of Civil and Enviromental Engineering. 